\title{
NONCOMMUTATIVE JORDAN RINGS
}

\author{
BY \\ KEVIN MCCRIMMON
}

\begin{abstract}
Heretofore most investigations of noncommutative Jordan algebras have been restricted to algebras over fields of characteristic $\neq 2$ in order to make use of the passage from a noncommutative Jordan algebra $\mathfrak{A}$ to the commutative Jordan algebra $\mathfrak{A}^{+}$with multiplication $x \cdot y=\frac{1}{2}(x y+y x)$.

We have recently shown that from an arbitrary noncommutative Jordan algebra $\mathfrak{A}$ one can construct a quadratic Jordan algebra $\mathfrak{A}^{+}$with a multiplication $U_{x} y$ $=x(x y+y x)-x^{2} y=(x y+y x) x-y x^{2}$, and that these quadratic Jordan algebras have a theory analogous to that of commutative Jordan algebras.

In this paper we make use of this passage from $\mathfrak{A}$ to $\mathfrak{U}^{+}$to derive a general structure theory for noncommutative Jordan rings. We define a Jacobson radical and show it coincides with the nil radical for rings with descending chain condition on inner ideals; semisimple rings with d.c.c. are shown to be direct sums of simple rings, and the simple rings to be essentially the familiar ones.

In addition we obtain results, which seem to be new even in characteristic $\neq 2$, concerning algebras without finiteness conditions. We show that an arbitrary simple noncommutative Jordan ring containing two nonzero idempotents whose sum is not 1 is either commutative or quasiassociative.
\end{abstract}

1. Axioms and basic results. Throughout this paper $\mathfrak{A}$ will denote a noncommutative Jordan algebra over a unital, commutative, associative ring of scalars $\Phi$; we obtain Jordan rings by taking $\Phi=Z$, the ring of integers, and the usual linear algebras by taking $\Phi$ to be a field. Unless otherwise stated, $\Phi$ will be completely arbitrary, and we impose no finiteness conditions on $\mathfrak{A}$.

We recall the definition [9, p. 472], [11, p. 141]. A noncommutative Jordan algebra $\mathfrak{A}$ is a space together with a bilinear product $x y$ such that the flexible law

$$
[x, y, x]=0 \quad([x, y, z]=(x y) z-x(y z))
$$

and the Jordan identity

$$
\left[x^{2}, y, x\right]=0
$$

hold strictly, i.e. they remain valid in any scalar extension $\mathfrak{A}_{\Omega}=\mathfrak{A} \otimes_{\Phi} \Omega$. This will automatically be the case if these identities hold in $\mathfrak{A}$ and $\Phi$ is a field with at least three elements (as in most previous investigations), and in general it merely amounts to assuming in addition the linearized version

$$
\left[x^{2}, y, z\right]+[x \circ z, y, x]=0 \quad(x \circ y=x y+y x) .
$$

Received by the editors June 25, 1970.

AMS 1969 subject classifications. Primary 1740.

Key words and phrases. Noncommutative Jordan algebra, quadratic Jordan algebra, idempotent, radical, inner ideal. 
These conditions are equivalent to assuming that for any $x$ the left and right multiplications $L_{x}, R_{x}, L_{x^{2}}, R_{x^{2}}$ all commute (and that this continues to hold in all extensions). We write this as

$$
\left[E_{x}, F_{x}\right]=\left[E_{x^{2}}, F_{x}\right]=0 \quad(E, F=L, R) .
$$

These results are well known when $\frac{1}{2} \in \Phi[1$, p. 574], and carry over in general using the operator form $\left[L_{x}, R_{x}\right]=0$ and $\left[L_{x^{2}}, R_{x}\right]=0$ of (1) and (2) together with the linearization

$$
[x, y, z]+[z, y, x]=0 .
$$

From any noncommutative Jordan algebra $\mathfrak{A}$ we can construct a quadratic Jordan algebra $\mathfrak{Q}^{+}$with operations

$$
x^{2}=x x, \quad U_{x} y=x(x y+y x)-x^{2} y=(x y+y x) x-y x^{2}
$$

[7, p. 276]. Thus the $U$-operators of $\mathfrak{A}^{+}$are formed from the multiplications in $\mathfrak{A}$ by

$$
U_{x}=L_{x} V_{x}-L_{x^{2}}=R_{x} V_{x}-R_{x^{2}} \quad\left(V_{x}=L_{x}+R_{x}\right) .
$$

A basic property of these operators is that by (3) $U_{x}$ commutes with $L_{x}, R_{x}, L_{x^{2}}$, $R_{x^{2}}$. (For a detailed exposition of quadratic Jordan algebras, see [13].)

We can polarize the cubic composition to obtain a trilinear composition

$$
\left\{\begin{array}{lll}
x y & z
\end{array}\right\}=U_{x, z} y, \quad U_{x, z}=U_{x+z}-U_{x}-U_{z}
$$

and from this another operator

$$
V_{x, y} z=\left\{\begin{array}{lll}
x & y & z
\end{array} .\right.
$$

Further useful formulas involving the $U$-operators are

$$
\begin{aligned}
x\left(U_{y} x\right) & =\left(U_{x} y\right) y, & y \circ U_{x} y & =x \circ U_{y} x, \\
{\left[R_{z}, U_{x}\right] } & =L_{x} V_{z, x}-V_{x, z} L_{x}, & {\left[L_{z}, U_{x}\right] } & =R_{x} V_{z, x}-V_{x, z} R_{x}, \\
U_{x, x y} & =R_{y} U_{x}+U_{x} L_{y}, & U_{y x, x} & =L_{y} U_{x}+U_{x} R_{y} .
\end{aligned}
$$

To prove (6),

$$
\begin{array}{rlr}
\left(U_{x} y\right) y & =\left\{x(x \circ y)-x^{2} y\right\} y=R_{y} R_{x y y} x-\left(x^{2} y\right) y & \\
& =\left\{R_{x y} R_{y}-\left[R_{x}, R_{y^{2}}\right]\right\} x-\left(x^{2} y\right) y & \\
& =(x y)(x \circ y)-\left(x y^{2}\right) x+x^{2} y^{2}-\left(x^{2} y\right) y & \\
& =-\left[x^{2}, y, y\right]-x y^{2} x+[x, y, x \circ y]+x\{y(x \circ y)\} & \\
& =+[x \circ y, y, x]+[x, y, x \circ y]+x\left\{y(x \circ y)-y^{2} x\right\} & (\text { by (2)') } \\
& =x\left(U_{y} x\right) &
\end{array}
$$


The second part follows by interchanging $x$ and $y$ and adding. For (7),

$$
\begin{aligned}
\left\{\left[R_{z}, U_{x}\right]+\right. & \left.V_{x, z} L_{x}-L_{x} V_{z, x}\right\} y \\
= & \left(U_{x} y\right) z-U_{x}(y z)+\{x z x y\}-x\{z x y\} \\
= & \left(x \circ x y-x^{2} y\right) z-\left(x(x \circ y z)-x^{2}(y z)\right)+x(x y \circ z)+x y(x \circ z) \\
& -(x \circ x y) z-x(y(x \circ z)+z(x \circ y)-(y \circ z) x) \\
= & -\left[x^{2}, y, z\right]+[x, y, x \circ z]+x\{-x \circ y z+x y \circ z-z(x \circ y)+(y \circ z) x\} \\
= & {[x \circ z, y, x]+[x, y, x \circ z]+x\{-x(y z)+(x y) z-z(y x)+(z y) x\} } \\
= & x\{[x, y, z]+[z, y, x]\}=0
\end{aligned}
$$

and dually, while for (8)

$$
\begin{aligned}
U_{x, x y} z & =\left\{\begin{array}{lll}
x & z & x y
\end{array}\right\}=V_{x, z} L_{x} y \\
& =\left\{L_{x} V_{z, x}-\left[R_{z}, U_{x}\right]\right\} y \\
& =x\left\{\begin{array}{lll}
z & x & y
\end{array}-\left(U_{x} y\right) z+U_{x}(y z)\right. \\
& =\left(U_{x} z\right) y+U_{x}(y z) \\
& =\left\{R_{y} U_{x}+U_{x} L_{y}\right\} z
\end{aligned}
$$

and dually.

We define the powers $x^{n}$ recursively from the left by

$$
x^{1}=x, \quad x^{n+1}=x x^{n} .
$$

Note that the quadratic Jordan powers $\left(x^{1}=x, x^{2}=x^{2}, x^{n+2}=U_{x} x^{n}\right)$ coincide with the noncommutative powers; clearly $x^{n}$ has the same meaning in both, for $n=1,2$, while, for $n=3, U_{x} x=x(x x+x x)-x^{2} x=2 x x^{2}-x^{2} x=x x^{2}=x^{3}$ by (1), and if true for $n+2$ then, for $n+3, U_{x} x^{n+1}=U_{x} L_{x} x^{n}=L_{x} U_{x} x^{n}=L_{x} x^{n+2}=x^{n+3}$. Throughout the paper we will see examples of this close relation between properties of $\mathfrak{A}$ and properties of $\mathfrak{U}^{+}$.

As a generalization of (3) we have

All $L_{x^{n}}, R_{x^{n}}$ belong to the commutative algebra of linear operators generated by $L_{x}, R_{x}, U_{x}$.

This is clear for $n=1,2$ by (5), while for $n+2$ we have $L_{x^{n+2}} y=R_{y} U_{x} x^{n}$ $=\left\{U_{x y, x}-U_{x} L_{y}\right\} x^{n}$ (by (8)) $=\left\{x y x^{n} x\right\}-U_{x} R_{x^{n}} y$ so that $L_{x^{n+2}}=V_{x, x^{n}} L_{x}-U_{x} R_{x^{n}}$ is generated by $L_{x}, R_{x}, U_{x}$ by induction (note $V_{x, x^{n}}=V_{x^{n+1}}$ in any quadratic Jordan algebra).

Any noncommutative Jordan algebra is strictly power-associative in the sense that

$$
x^{n} x^{m}=x^{n+m}
$$

holds in $\mathfrak{A}$ and any extension of $\mathfrak{A}[9, \mathrm{p} .473]$. This follows for $n=1$ by definition, and by induction $x^{n+1} x^{m}=R_{x^{m}} L_{x} x^{n}=L_{x} R_{x^{m}} x^{n}$ (using (9)) $=L_{x} x^{n+m}=x^{n+m+1}$. 
If $\frac{1}{2} \in \Phi$, R. D. Schafer proved [9, p. 473] that a flexible algebra was a noncommutative Jordan algebra if and only if it was "Jordan admissible" in the sense that $\mathfrak{A}^{+}$was a commutative Jordan algebra. Unfortunately, this result does not extend to arbitrary $\Phi$. Of course, any noncommutative Jordan algebra $\mathfrak{A}$ is flexible and "Jordan admissible" in the sense that $\mathfrak{A}^{+}$(with the $U$-operators defined as in (4)) is a quadratic Jordan algebra. However, flexibility and Jordan-admissibility are not enough to guarantee (2); an example is $\mathfrak{A}=\mathfrak{F} / \mathfrak{A}$ where $\mathfrak{F}$ is the free nonassociative algebra and $\mathfrak{R}$ the ideal generated by all $x y+y x$ and all monomials of degree $\geqq 5$ (cf. the axioms of [7, p. 271]). Of course $2 x^{2}=x x+x x=0$, so if $\frac{1}{2} \in \Phi$ the Jordan identity would be trivial, but for $\Phi$ of characteristic 2 the identity (2) fails even though the algebra is flexible (even commutative) and Jordan-admissible.

2. Units and inverses. A unit element 1 satisfies the usual conditions $1 x=x 1=x$. If 1 is a unit for $\mathfrak{A}$ it is also one for $\mathfrak{A}^{+}$since $U_{x} 1=x(x 1+1 x)-x^{2} 1=x^{2}$ and $U_{1} x$ $=1(1 x+x 1)-1 x=x\left[7\right.$, p. 273]. Conversely, if 1 is a unit for $\mathfrak{A}^{+}$then $V_{1}=2 I$, $U_{1}=I$, and $1^{2}=1$, plus $I=U_{1}=L_{1} V_{1}-L_{1}^{2}=R_{1} V_{1}-R_{1}{ }^{2}$, imply $I=L_{1}=R_{1}$ and thus 1 is a unit for $\mathfrak{A}$. We may adjoin a unit to any noncommutative Jordan algebra $\mathfrak{A}$ in the usual manner to obtain a unital noncommutative Jordan algebra $\mathfrak{A}^{\prime}=\Phi 1+\mathfrak{A}$; we have a similar process for quadratic Jordan algebras, and $\left\{\mathfrak{A}^{\prime}\right\}^{+}=\left\{\mathfrak{A}^{+}\right\}^{\prime}$.

An element $x$ of a unital noncommutative Jordan algebra $\mathfrak{A}$ is invertible with inverse $y$ if

$$
x y=y x=1, \quad x^{2} y=y x^{2}=x .
$$

Then $x$ and $y$ are also inverses in $\mathfrak{A}^{+}$(i.e. $U_{y} x=y, U_{y} x^{2}=1$ ) since $U_{y} x^{2}$ $=y\left(y x^{2}+x^{2} y\right)-y^{2} x^{2}=y\left(x^{2} y\right)-\left[y, y, x^{2}\right]=y x+[x, y, x y+y x]\left(\right.$ by $\left.(2)^{\prime},(1)^{\prime}\right)=y x$ $=1$ and $U_{y} x=U_{y} L_{y} x^{2}=L_{y} U_{y} x^{2}=L_{y} 1=y$. Conversely, if $x$ and $y$ are inverses in $\mathfrak{A}^{+}$(so that $U_{x}$ is necessarily invertible) they are inverses in $\mathfrak{A}$ because $U_{x} y=x$, $U_{x} y^{2}=1$ imply $U_{x} E_{x} y=E_{x} U_{x} y=E_{x} x=x^{2}=U_{x} 1, \quad U_{x} E_{x^{2}} y=E_{x^{2}} U_{x} y=E_{x^{2}} x=x^{3}$ $=U_{x} x$ for $E=L, R$ by (9), so cancelling $U_{x}$ yields $x y=y x=1, x^{2} y=y x^{2}=x$. Thus the notions of invertibility in $\mathfrak{A}$ and $\mathfrak{A}^{+}$coincide.

Writing $x^{-1}$ for the (unique) inverse of $x$, we claim

$$
L_{x^{-1}}=U_{x}^{-1} R_{x}, \quad R_{x^{-1}}=U_{x}^{-1} L_{x} .
$$

For example, $U_{x} L_{x}-1 y=U_{x} R_{y} x^{-1}=\left\{U_{y x, x}-L_{y} U_{x}\right\} x^{-1}$ (by (8)) $=\left\{y x \times x^{-1}\right\}$ $-y\left\{U_{x} x^{-1}\right\}=2 y x-y x=y x=R_{x} y$ since $\left\{z x x^{-1}\right\}=2 z$ in any quadratic Jordan algebra (e.g. see [14]).

From this we also see that if we define negative powers by $x^{-n}=\left(x^{-1}\right)^{n}$ then from (9), (5), (11) we obtain

$$
\begin{aligned}
& \text { All } L_{x^{n}}, R_{x^{n}} \text { belong to the commutative algebra of linear operators } \\
& \text { generated by } L_{x}, R_{x}, U_{x}, U_{x}^{-1} \text {. }
\end{aligned}
$$

These results improve on those of [6, pp. 943-944]. From this we could extend (10) to include negative powers. 
3. Idempotents and Peirce decompositions. An idempotent in $\mathfrak{A}$ is an element $e$ with $e^{2}=e$. Two idempotents $e, f$ are orthogonal if $e f=f e=0$. In this case $U_{e} f$ $=e(e f+f e)-e^{2} f=0, e \circ f=e f+f e=0, U_{f} e=0$ so $e, f$ are orthogonal in $\mathfrak{A}^{+}$. Conversely, if $U_{e} f=e \circ f=U_{f} e=0$ in $\mathfrak{A}^{+}$then $e f=e^{2} f=\left\{L_{e} V_{e}-U_{e}\right\} f=0, f e=0$, and $e, f$ are orthogonal in $\mathfrak{A}$. Thus the notions of idempotence and orthogonality coincide in $\mathfrak{A}$ and $\mathfrak{A}^{+}$.

An idempotent $e$ determines a Peirce decomposition $\mathfrak{A}^{+}=\mathfrak{A}_{1} \oplus \mathfrak{A}_{1 / 2} \oplus \mathfrak{A}_{0}$ of $\mathfrak{A}^{+}$into a direct sum of subspaces. Here $U_{e}=I, V_{e}=2 I$ on $\mathfrak{A}_{1} ; U_{e}=0, V_{e}=I$ on $\mathfrak{A}_{1 / 2} ; U_{e}=V_{e}=0$ on $\mathfrak{A}_{0}$. We claim $L_{e}=R_{e}=i I$ on $\mathfrak{A}_{i}$ for $i=1,0$ (in general we cannot say anything about the action of $L_{e}$ on $\left.\mathfrak{A}_{1 / 2}\right)$; ex=xe=ix for $x \in \mathfrak{H}_{\mathfrak{i}}$. This follows since $i I=U_{e}=L_{e} V_{e}-L_{e}=L_{e}\left(V_{e}-I\right)=(2 i-1) L_{e}$ on $\mathfrak{A}_{i}$, and similarly for $R_{e}$.

Peirce Decomposition Theorem. If $e$ is an idempotent in a noncommutative Jordan algebra $\mathfrak{A}$ then $\mathfrak{A}$ is the direct sum $\mathfrak{A}=\mathfrak{A}_{1} \oplus \mathfrak{A}_{1 / 2} \oplus \mathfrak{A}_{0}$ of subspaces

$$
\mathfrak{A}_{1}=\{x \mid e x=x e=x\}, \quad \mathfrak{A}_{1 / 2}=\{x \mid e x+x e=x\}, \quad \mathfrak{A}_{0}=\{x \mid e x=x e=0\} .
$$

These coincide with the Peirce spaces $\mathfrak{A}_{1}=U_{e} \mathfrak{A}, \mathfrak{A}_{1 / 2}=U_{e, 1-e} \mathfrak{A}, \mathfrak{A}_{0}=U_{1-e} \mathfrak{A}$ of $\mathfrak{A}^{+}$.

Peirce Relations Theorem. The Peirce spaces multiply according to

(PR 1) $\mathfrak{A}_{i}^{2} \subset \mathfrak{A}_{i}(i=0,1)$,

(PR 2) $\mathfrak{A}_{i} \mathfrak{A}_{1 / 2}+\mathfrak{A}_{1 / 2} \mathfrak{A}_{i} \subset \mathfrak{A}_{1 / 2}(i=0,1)$,

(PR 3) $x \in \mathfrak{A}_{1 / 2} \Rightarrow x^{2} \in \mathfrak{A}_{1}+\mathfrak{A}_{0}$,

(PR 4) $\mathfrak{A}_{1} \mathfrak{A}_{0}=\mathfrak{A}_{0} \mathfrak{A}_{1}=0$.

Proof. (PR 3) follows from the corresponding result in $\mathfrak{A}^{+}$(or it can be shown directly). For the others we use the basic commutativity formula

$$
\left[E_{x}, F_{e}\right]=0 \quad\left(E, F=L, R, x \in \mathfrak{A}_{1}+\mathfrak{A}_{0}\right)
$$

which follows from $0=\left[E_{x}, F_{e^{2}}\right]+\left[E_{e}, F_{x_{\circ} e}\right]$ (linearizing (3)) $=\left[E_{x}, F_{e}\right]+2 i\left[E_{e}, F_{x}\right]$ $=(1-2 i)\left[E_{x}, F_{e}\right]$ (linearizing (3) again) when $x \in \mathfrak{A}_{i}$.

This shows multiplications by $\mathfrak{A}_{0}$ or $\mathfrak{A}_{1}$ commute with the Peirce projections built up from $L_{e}$ and $R_{e}$, hence leave the spaces $\mathfrak{A}_{j}$ invariant, which establishes (PR 1), (PR 2), and shows $\mathfrak{A}_{1} \mathfrak{A}_{0} \subset \mathfrak{A}_{1} \cap \mathfrak{A}_{0}=0$. Similarly $\mathfrak{A}_{0} \mathfrak{A}_{1}=0$, whence (PR 4).

We have the following extremely important formulas [3, p. 189]. They allow us to recover certain products $x y$ in $\mathfrak{A}$ from the products $x \circ y$ in $\mathfrak{A}^{+}$together with multiplication by $e$. If $x_{i}$ belong to the Peirce spaces $\mathfrak{A}_{i}$ relative to $e$,

$$
\begin{gathered}
e\left(x_{1 / 2} \circ x_{0}\right)=e x_{1 / 2} \circ x_{0}=x_{1 / 2} x_{0}, \quad\left(x_{1 / 2} \circ x_{0}\right) e=x_{1 / 2} e \circ x_{0}=x_{0} x_{1 / 2}, \\
e\left(x_{1 / 2} \circ x_{1}\right)=e x_{1 / 2} \circ x_{1}=x_{1} x_{1 / 2}, \quad\left(x_{1 / 2} \circ x_{1}\right) e=x_{1 / 2} e \circ x_{1}=x_{1 / 2} x_{1}, \\
P_{0}\left(x_{1 / 2} \circ e y_{1 / 2}\right)=P_{0}\left(x_{1 / 2} e \circ y_{1 / 2}\right)=P_{0}\left(x_{1 / 2} y_{1 / 2}\right), \\
P_{1}\left(e x_{1 / 2} \circ y_{1 / 2}\right)=P_{1}\left(x_{1 / 2} \circ y_{1 / 2} e\right)=P_{1}\left(x_{1 / 2} y_{1 / 2}\right),
\end{gathered}
$$


where the $P_{i}$ are the Peirce projections on $\mathfrak{A}_{\mathfrak{i}}$. In particular, we have the following corollaries:

(14)' If $\mathfrak{B}_{1 / 2}$ is a subspace of $\mathfrak{2}_{1 / 2}$ satisfying $e \mathfrak{B}_{1 / 2} \subset \mathfrak{B}_{1 / 2}$, and $\mathfrak{B}_{i}$ any subspace of $\mathfrak{A}_{i}$ for $i=1,0$, then

$$
\mathfrak{B}_{i} \mathfrak{B}_{1 / 2}+\mathfrak{B}_{1 / 2} \mathfrak{B}_{i}=\mathfrak{B}_{i} \circ \mathfrak{B}_{1 / 2} .
$$

(15)' If $\mathfrak{B}_{1 / 2}$ is any subspace of $\mathfrak{A}_{1 / 2}$, then for $i=1,0$,

$$
P_{i}\left(\mathfrak{A}_{1 / 2} \mathfrak{B}_{1 / 2}+\mathfrak{B}_{1 / 2} \mathfrak{A}_{1 / 2}\right)=P_{i}\left(\mathfrak{U}_{1 / 2} \circ \mathfrak{B}_{1 / 2}\right) .
$$

For example, to prove the second part of (14) we write $x_{1} x_{1 / 2}=\left[R_{x_{1 / 2}}, U_{e}\right] x_{1}$ $=\left\{L_{e} V_{x_{1 / 2}, e}-V_{e, x_{1,2}} L_{e}\right\} x_{1}=e\left\{x_{1 / 2}\right.$ e $\left.x_{1}\right\}-\left\{e x_{1 / 2} x_{1}\right\}=e\left(x_{1 / 2} \circ x_{1}\right)$ from the Peirce relation in $\mathfrak{A}^{+}\left[2\right.$, p. 1074] and (7). In (15) we use (8) to see $P_{1}\left(e x_{1 / 2} \circ y_{1 / 2}\right)$ $=\left\{e x_{1 / 2} y_{1 / 2} e\right\}=U_{e x_{1 / 2}, e} y_{1 / 2}=\left\{R_{x_{1 / 2}} U_{e}+U_{e} L_{x_{1 / 2}}\right\} y_{1 / 2}=P_{1}\left(x_{1 / 2} y_{1 / 2}\right)$. The other parts follow in the same way.

These allow us to improve on (13), namely

$$
\left[E_{x_{1}}, F_{y_{0}}\right]=0 \quad(E, F=L, R) .
$$

This is trivial on $\mathfrak{A}_{0}+\mathfrak{A}_{1}$ by (PR 4). On $\mathfrak{A}_{1 / 2},\left[E_{x_{1}}, F_{y_{0}}\right]=\left[V_{x_{1}} E_{e}, V_{y_{0}} G_{e}\right]$ (by (14), for $G$ the opposite of $F)=\left[V_{x_{1}}, V_{y_{0}}\right] E_{e} G_{e}\left(\right.$ by (13)) $=0$ since $V_{\mathfrak{A}_{0}}$ and $V_{\mathfrak{A}_{1}}$ commute in any quadratic Jordan algebra.

More esoteric are the formulas

$$
\begin{gathered}
z_{i} \circ P_{1 / 2}\left(x_{1 / 2} y_{1 / 2}\right)=P_{1 / 2}\left\{x_{1 / 2}\left(z_{i} \circ y_{1 / 2}\right)\right\}=P_{1 / 2}\left\{\left(z_{i} \circ x_{1 / 2}\right) y_{1 / 2}\right\} \quad(i=1,0) \\
P_{i}\left\{x_{1 / 2} \circ P_{1 / 2}\left(y_{1 / 2} z_{1 / 2}\right)\right\}=P_{i}\left\{z_{1 / 2} \circ P_{1 / 2}\left(x_{1 / 2} y_{1 / 2}\right)\right\} \\
=P_{i}\left\{y_{1 / 2} \circ P_{1 / 2}\left(z_{1 / 2} x_{1 / 2}\right)\right\} \quad(i=1,0) \\
U_{x_{1 / 2}} y_{i}=P_{\{}\left\{x_{1 / 2}\left(x_{1 / 2} \circ y_{i}\right)\right\}=P_{j}\left\{\left(y_{i} \circ x_{1 / 2}\right) x_{1 / 2}\right\} \quad(j=1-i) \\
x_{1 / 2}^{2} y_{i}=P_{i}\left\{x_{1 / 2}\left(x_{1 / 2} \circ y_{i}\right)\right\}, \quad y_{i} x_{1 / 2}^{2}=P_{i}\left\{\left(y_{i} \circ x_{1 / 2}\right) x_{1 / 2}\right\} \\
x_{1 / 2}^{2} \circ y_{i}=P_{i}\left\{x_{1 / 2} \circ\left(x_{1 / 2} \circ y_{i}\right)\right\} \\
{\left[x_{i}, y_{i}, z_{1 / 2}\right]=y_{i}\left(z_{1 / 2} x_{i}\right)=\left(x_{i} z_{1 / 2}\right) y_{i},} \\
{\left[z_{1 / 2}, y_{i}, x_{i}\right]=-\left(x_{i} z_{1 / 2}\right) y_{i}=-y_{i}\left(z_{1 / 2} x_{i}\right)}
\end{gathered}
$$

To prove (17), let $x_{1 / 2} y_{1 / 2}=a_{1}+a_{1 / 2}+a_{0}, y_{1 / 2} x_{1 / 2}=b_{1}+b_{1 / 2}+b_{0}$ (so $b_{1 / 2}=-a_{1 / 2}$ by (PR 3)). Then

$$
\begin{aligned}
0 & =P_{1 / 2}\left\{\left[x_{1 / 2}, y_{1 / 2}, z_{i}\right]+\left[z_{i}, y_{1 / 2}, x_{1 / 2}\right]\right\} \\
& =P_{1 / 2}\left\{\left(x_{1 / 2} y_{1 / 2}\right) z_{i}-x_{1 / 2}\left(y_{1 / 2} z_{i}\right)+\left(z_{i} y_{1 / 2}\right) x_{1 / 2}-z_{i}\left(y_{1 / 2} x_{1 / 2}\right)\right\} \\
& =P_{1 / 2}\left\{a_{1 / 2} z_{i}-x_{1 / 2}\left(y_{1 / 2} z_{i}\right)+\left(z_{i} \circ y_{1 / 2}\right) x_{1 / 2}-\left(y_{1 / 2} z_{i}\right) x_{1 / 2}+z_{i} a_{1 / 2}\right\} \\
& =P_{1 / 2}\left\{a_{1 / 2} \circ z_{i}-x_{1 / 2} \circ\left(y_{1 / 2} z_{i}\right)+\left(z_{i} \circ y_{1 / 2}\right) x_{1 / 2}\right\} \\
& =a_{1 / 2} \circ z_{i}+P_{1 / 2}\left\{\left(z_{i} \circ y_{1 / 2}\right) x_{1 / 2}\right\}=a_{1 / 2} \circ z_{i}-P_{1 / 2}\left\{x_{1 / 2}\left(z_{i} \circ y_{1 / 2}\right)\right\} \quad \text { (by (PR 3)) }
\end{aligned}
$$


so $z_{i} \circ P_{1 / 2}\left(x_{1 / 2} y_{1 / 2}\right)=z_{i} \circ a_{1 / 2}=P_{1 / 2}\left\{x_{1 / 2}\left(z_{i} \circ y_{1 / 2}\right)\right\}$. This gives the first part of (17); the second follows by a similar argument (or note $z_{i} \circ P_{1 / 2}\left(x_{1 / 2} y_{1 / 2}\right)=$ $\left.-z_{i} \circ P_{1 / 2}\left(y_{1 / 2} x_{1 / 2}\right)=-P_{1 / 2}\left\{y_{1 / 2}\left(z_{i} \circ x_{1 / 2}\right)\right\}=P_{1 / 2}\left\{\left(z_{i} \circ x_{1 / 2}\right) y_{1 / 2}\right\}\right)$. For (18) we have

$$
\begin{aligned}
0= & P_{i}\left\{\left[z_{1 / 2}, x_{1 / 2}, y_{1 / 2}\right]+\left[y_{1 / 2}, x_{1 / 2}, z_{1 / 2}\right]\right\} \\
= & P_{i}\left\{\left(z_{1 / 2} x_{1 / 2}\right) y_{1 / 2}-z_{1 / 2}\left(x_{1 / 2} y_{1 / 2}\right)+\left(y_{1 / 2} x_{1 / 2}\right) z_{1 / 2}-y_{1 / 2}\left(x_{1 / 2} z_{1 / 2}\right)\right\} \\
= & P_{i}\left\{\left(z_{1 / 2} x_{1 / 2}\right) y_{1 / 2}-z_{1 / 2}\left(x_{1 / 2} y_{1 / 2}\right)+\left(x_{1 / 2} \circ y_{1 / 2}\right) z_{1 / 2}-\left(x_{1 / 2} y_{1 / 2}\right) z_{1 / 2}\right. \\
& \left.-y_{1 / 2}\left(x_{1 / 2} \circ z_{1 / 2}\right)+y_{1 / 2}\left(z_{1 / 2} x_{1 / 2}\right)\right\} \\
= & P_{i}\left\{\left(z_{1 / 2} x_{1 / 2}\right) \circ y_{1 / 2}-z_{1 / 2} \circ\left(x_{1 / 2} y_{1 / 2}\right)\right\}
\end{aligned}
$$

so $P_{\{}\left\{z_{1 / 2} \circ P_{1 / 2}\left(x_{1 / 2} y_{1 / 2}\right)\right\}=P_{i}\left\{y_{1 / 2} \circ P_{1 / 2}\left(z_{1 / 2} x_{1 / 2}\right)\right\}$; permuting cyclically gives (18). (19)-(20) follow from $U_{x_{1 / 2}} y_{i}=x_{1 / 2}\left(x_{1 / 2} \circ y_{i}\right)-x_{1 / 2}^{2} y_{i}=\left(y_{i} \circ x_{1 / 2}\right) x_{1 / 2}-y_{i} x_{1 / 2}^{2}$ by applying $P_{i}$ and $P_{j}$, noting $U_{x_{1 / 2}} y_{i} \in \mathfrak{A}_{j}$ and $y_{i} x_{1 / 2}^{2}, x_{1 / 2}^{2} y_{i} \in \mathfrak{A}_{i}$. (21) follows as in $[3,(12)$ on p. 189].

The most general decomposition is

General Peirce Decomposition. If $e_{1}, \ldots, e_{n}$ are pairwise orthogonal idempotents then

$$
\mathfrak{U}=\sum \bigoplus_{i, j=0}^{n} \mathfrak{A}_{i j}
$$

where, for $i, j, 0 \neq$,

$$
\begin{aligned}
\mathfrak{U}_{i i} & =\left\{x \mid e_{i} x=x e_{i}=x\right\}, \quad \mathfrak{U}_{i j}=\left\{x \mid e_{i} x+x e_{i}=e_{j} x+x e_{j}=x\right\}=\mathfrak{A}_{j i}, \\
\mathfrak{A}_{00} & =\left\{x \mid e_{i} x=x e_{i}=0 \text { for all } i\right\}, \\
\mathfrak{A}_{i 0} & =\left\{x \mid e_{i} x+x e_{i}=x, e_{j} x=x e_{j}=0 \text { for all } j \neq i\right\}=\mathfrak{A}_{0 i} .
\end{aligned}
$$

This coincides with the Peirce decomposition of $\mathfrak{A}^{+}$relative to the $e_{i}: \mathfrak{A}_{\mathfrak{i}}=U_{e_{\mathfrak{a}}} \mathfrak{A}$, $\mathfrak{A}_{i j}=U_{e_{i}, e_{j}} \mathfrak{A}, \mathfrak{A}_{00}=U_{1-e} \mathfrak{A}, \mathfrak{A}_{i 0}=U_{1-e, e_{i}} \mathfrak{A}$ for $e=\sum e_{i}$. For $x \in \mathfrak{A}_{i j}$ we have $e_{i} x=x e_{j}$, $e_{k} x=x e_{k}=0(k \neq i, j)$. (The last statement follows because $e_{i} x=x-x e_{i}=x e_{j}$ as $e_{i}+e_{j}$ is the unit for $\mathfrak{A}_{i \mathfrak{i}}+\mathfrak{A}_{i j}+\mathfrak{A}_{j j}$.)

General Peirce Relations. The Peirce subspaces multiply according to

(GPR 1) $\mathfrak{A}_{\mathfrak{i i}}^{2} \subset \mathfrak{A}_{\mathfrak{i n}}$,

(GPR 2) $\mathfrak{A}_{i i} \mathfrak{A}_{i j}+\mathfrak{A}_{i j} \mathfrak{A}_{i i} \subset \mathfrak{A}_{i j}$,

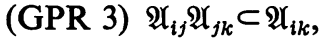

(GPR 4) $\mathfrak{A}_{i j}^{2} \subset \mathfrak{U}_{i \mathfrak{i}}+\mathfrak{A}_{i j}+\mathfrak{U}_{j j}$,

(GPR 5) $x \in \mathfrak{A}_{i j} \Rightarrow x^{2} \in \mathfrak{A}_{i i}+\mathfrak{A}_{j j}$,

where $\mathfrak{A}_{i j} \mathfrak{A}_{k l}=0$ if there are no shared indices. Furthermore, the general Peirce spaces may be expressed as

$$
\mathfrak{A}_{i \mathfrak{i}}=\mathfrak{A}_{1}\left(e_{i}\right), \quad \mathfrak{A}_{i j}=\mathfrak{A}_{1 / 2}\left(e_{i}\right) \cap \mathfrak{U}_{1 / 2}\left(e_{j}\right)
$$


in terms of the Peirce spaces correspending to the individual idempotents. If $J$ is any subset of the index set $I$ and $e_{J}=\sum_{j \in J} e_{j}$ then the Peirce spaces of $e_{J}$ are

$$
\begin{gathered}
\mathfrak{A}_{1}\left(e_{J}\right)=\sum_{j, j^{\prime} \in J} \mathfrak{A}_{j j^{\prime}}, \quad \mathfrak{A}_{1 / 2}\left(e_{J}\right)=\sum_{j \in J ; i \notin J} \mathfrak{A}_{i j}, \\
\mathfrak{A}_{0}\left(e_{J}\right)=\sum_{i, i^{\prime} \in J} \mathfrak{A}_{i i^{\prime}} .
\end{gathered}
$$

The multiplication rules can be proved as in the case of a single idempotent since the $F_{x}\left(x \in \mathfrak{A}_{k k}\right)$ commute with all Peirce projections $P_{i j}$ and the $F_{x}\left(x \in \mathfrak{A}_{j k}\right)$ commute with the Peirce projections relative to $e_{j}+e_{k}$. Alternatively, the rules can be deduced from the case of a single idempotent, noting that $\mathfrak{A}_{i i}=\mathfrak{A}_{1}\left(e_{i}\right)$; $\mathfrak{A}_{i i}=\mathfrak{U}_{1}\left(e_{i}\right) \subset \mathfrak{U}_{0}\left(e_{j}\right), \quad \mathfrak{U}_{i j}=\mathfrak{U}_{1 / 2}\left(e_{i}\right) \cap \mathfrak{A}_{1 / 2}\left(e_{j}\right) ; \quad \mathfrak{U}_{i j} \subset \mathfrak{U}_{1 / 2}\left(e_{i}\right) \cap \mathfrak{U}_{0}\left(e_{k}\right), \quad \mathfrak{A}_{j k} \subset \mathfrak{U}_{0}\left(e_{i}\right)$ $\cap \mathfrak{A}_{1 / 2}\left(e_{k}\right) ; \mathfrak{A}_{i j} \subset \mathfrak{A}_{1}\left(e_{i}+e_{j}\right)$ for (GPR 1-4) respectively.

The formula (20) can be improved to

$$
\begin{gathered}
x_{i j}^{2} y_{j k}=x_{i j}\left(x_{i j} \circ y_{j k}\right), \quad y_{j k} x_{i j}^{2}=\left(y_{j k} \circ x_{i j}\right) x_{i j}, \\
x_{i j}^{2} \circ y_{j k}=x_{i j} \circ\left(x_{i j} \circ y_{j k}\right),
\end{gathered}
$$

since $P_{j k}\left\{x_{i j}\left(x_{i j} \circ y_{j k}\right)\right\}=x_{i j}\left(x_{i j} \circ y_{j k}\right)$ by (GPR 3).

4. Ideal-building lemmas. In this section we use the Peirce relations to construct ideals. The most important construction is

PROPOSITION 1 [3, p. 189]. If $\mathfrak{B}_{1 / 2}$ is a subspace of $\mathfrak{A}_{1 / 2}$ such that $e \mathfrak{B}_{1 / 2} \subset \mathfrak{B}_{1 / 2}$ and $\mathfrak{A}_{i} \circ \mathfrak{B}_{1 / 2} \subset \mathfrak{B}_{1 / 2}(i=1,0)$ then $\mathfrak{B}_{i}=P_{i}\left\{\mathfrak{A}_{1 / 2} \circ \mathfrak{B}_{1 / 2}\right\}$ is an ideal in $\mathfrak{A}_{i} \cdot \mathfrak{B}=\mathfrak{B}_{1}+\mathfrak{B}_{1 / 2}+\mathfrak{B}_{0}$ will be an ideal in $\mathfrak{A}$ if

(i) $\mathfrak{A}_{1 / 2} \circ \mathfrak{B}_{i} \subset \mathfrak{B}_{1 / 2}$,

(ii) $P_{1 / 2}\left\{\mathfrak{U}_{1 / 2} \mathfrak{B}_{1 / 2}\right\} \subset \mathfrak{B}_{1 / 2}$,

in which case $\mathfrak{B}$ coincides with the ideal in $\mathfrak{A}^{+}$generated by $\mathfrak{B}_{1 / 2}$.

Proof. That $\mathfrak{B}_{i}$ is an ideal in $\mathfrak{A}_{i}$ follows from linearized (20). The condition that $\mathfrak{B}$ be an ideal, $\mathfrak{A} \mathfrak{B}+\mathfrak{B P} \subset \mathfrak{B}$, reduces to (i) and (ii) since $\mathfrak{A}_{i} \mathfrak{B}_{\mathfrak{i}}+\mathfrak{B}_{i} \mathfrak{A}_{\mathfrak{i}} \subset \mathfrak{B}_{i}$ by the first part, $\mathfrak{A}_{i} \mathfrak{B}_{j}=\mathfrak{B}_{j} \mathfrak{A}_{i}=0$ by orthogonality, $\mathfrak{A}_{i} \mathfrak{B}_{1 / 2}+\mathfrak{B}_{1 / 2} \mathfrak{A}_{i} \subset \mathfrak{B}_{1 / 2}$ by $(14)^{\prime}$ and our assumptions about $\mathfrak{B}_{1 / 2}$, while $P_{i}\left(\mathfrak{A}_{1 / 2} \mathfrak{B}_{1 / 2}+\mathfrak{B}_{1 / 2} \mathfrak{A}_{1 / 2}\right)=\mathfrak{B}_{i}$ by $(15)^{\prime}$ and the definition of the $\mathfrak{B}_{i}$.

Since the $\mathfrak{B}_{\mathfrak{i}}$ are generated from $\mathfrak{B}_{1 / 2}$ by Jordan products in $\mathfrak{A}^{+}, \mathfrak{B}$ is contained in the ideal of $\mathfrak{A}^{+}$generated by $\mathfrak{B}_{1 / 2}$, and if $\mathfrak{B}$ is an ideal in $\mathfrak{A}$ it is also one in $\mathfrak{A}^{+}$.

LEMMA 1. If $\mathfrak{B}_{1 / 2}=\mathfrak{A}_{1 / 2}$ and $\mathfrak{B}_{i}=P_{i}\left(\mathfrak{A}_{1 / 2} \circ \mathfrak{A}_{1 / 2}\right)$ then $\mathfrak{B}=\mathfrak{B}_{1}+\mathfrak{B}_{1 / 2}+\mathfrak{B}_{0}$ is an ideal in $\mathfrak{A}$ which coincides with the ideal in $\mathfrak{A}^{+}$generated by $\mathfrak{U}_{1 / 2}$.

LEMmA 2. If $\mathfrak{B}_{1 / 2}=\mathfrak{A}_{0} \circ \mathfrak{A}_{1 / 2}, \mathfrak{B}_{i}=P_{i}\left(\mathfrak{A}_{1 / 2} \circ \mathfrak{B}_{1 / 2}\right)$ then $\mathfrak{B}=\mathfrak{B}_{1}+\mathfrak{B}_{1 / 2}+\mathfrak{B}_{0}$ is an ideal in $\mathfrak{A}$.

Proof. Clearly $e \mathfrak{B}_{1 / 2} \subset \mathfrak{B}_{1 / 2}$ since $L_{e}$ commutes with $V_{\mathfrak{A}_{0}}$ by (13), $\mathfrak{A}_{0} \circ \mathfrak{B}_{1 / 2} \subset \mathfrak{B}_{1 / 2}$ is trivial, and $\mathfrak{A}_{1} \circ \mathfrak{B}_{1 / 2} \subset \mathfrak{B}_{1 / 2}$ because $V_{\mathfrak{A}_{0}}$ commutes with $V_{\mathfrak{A}_{1}}$ by (16). To see that $\mathfrak{B}_{1 / 2}$ satisfies (i) of Proposition 1, note $\mathfrak{A}_{1 / 2} \circ \mathfrak{B}_{0} \subset \mathfrak{B}_{1 / 2}$ is trivial and 
$\mathfrak{A}_{1 / 2} \circ \mathfrak{B}_{1}=\mathfrak{A}_{1 / 2} \circ P_{1}\left\{\mathfrak{U}_{1 / 2} \circ\left(\mathfrak{U}_{0} \circ \mathfrak{A}_{1 / 2}\right)\right\}=\mathfrak{A}_{1 / 2} \circ\left\{\mathfrak{A}_{1 / 2} \mathfrak{A}_{0} \mathfrak{A}_{1 / 2}\right\} \quad$ (linearized

$=-\mathfrak{A}_{0} \circ\left\{\mathfrak{A}_{1 / 2} \mathfrak{A}_{1 / 2} \mathfrak{A}_{1 / 2}\right\}+\left\{\mathfrak{A}_{0} \mathfrak{A}_{1 / 2} \mathfrak{A}_{1 / 2}\right\} \circ \mathfrak{A}_{1 / 2}+\left\{\mathfrak{A}_{0} \mathfrak{A}_{1 / 2} \mathfrak{A}_{1 / 2}\right\} \circ \mathfrak{A}_{1 / 2} \quad$ (linearizing (6)) $\subset \mathfrak{A}_{0} \circ \mathfrak{A}_{1 / 2}$ (by $\left[2\right.$, p. 1074]) $=\mathfrak{B}_{1 / 2}$. For (ii) note $P_{1 / 2}\left(\mathfrak{U}_{1 / 2} \mathfrak{B}_{1 / 2}\right)$ $=P_{1 / 2}\left\{\mathfrak{A}_{1 / 2}\left(\mathfrak{U}_{0} \circ \mathfrak{A}_{1 / 2}\right)\right\}=\mathfrak{A}_{0} \circ P_{1 / 2}\left(\mathfrak{A}_{1 / 2} \mathfrak{A}_{1 / 2}\right)$ (by (17)) $\subset \mathfrak{U}_{0} \circ \mathfrak{A}_{1 / 2}=\mathfrak{B}_{1 / 2}$. Thus $\mathfrak{B}_{1 / 2}$ satisfies the conditions of the proposition.

Lemma 3. For $i=1,0$ the space

$$
\mathfrak{Z}_{i}=\left\{z_{i} \in \mathfrak{A}_{i} \mid z_{i} \mathfrak{A}_{1 / 2}=\mathfrak{A}_{1 / 2} z_{i}=0\right\}=\left\{z_{i} \in \mathfrak{A}_{i} \mid z_{i} \circ \mathfrak{A}_{1 / 2}=0\right\}
$$

is an ideal in $\mathfrak{A}$.

Proof. Certainly $z_{i} \mathfrak{A}_{1 / 2}=\mathfrak{A}_{1 / 2} z_{i}=0$ implies $z_{i} \circ \mathfrak{A}_{1 / 2}=0$, and the converse follows from (14), so the two definitions of $B_{i}$ agree.

It suffices to show $\bigotimes_{i}$ is a left ideal. We have $\mathfrak{A}_{1 / 2} \mathbb{B}_{i}=0$ by definition and $\mathfrak{A}_{j} \mathbb{B}_{i}=0$ by orthogonality. For $\mathfrak{A}_{i} \mathcal{Z}_{i} \subset \mathfrak{Z}_{i}$ we use (21): $\left(a_{\mathfrak{i}} z_{i}\right) \mathfrak{A}_{1 / 2}=a_{i}\left(z_{i} \mathfrak{A}_{1 / 2}\right)+z_{i}\left(\mathfrak{A}_{1 / 2} a_{i}\right)=0$ and similarly $\mathfrak{A}_{1 / 2}\left(a_{i} z_{i}\right)=0$.

Now we investigate certain constructions of ideals from outer ideals. An outer ideal in $\mathfrak{A}$ is a subspace $\mathfrak{B}$ of $\mathfrak{A}$ such that $U_{\mathfrak{A}} \mathfrak{B} \subset \mathfrak{B}$, and an inner ideal if $U_{\mathfrak{B} \mathfrak{A}} \subset \mathfrak{B}$. (These concepts apply to arbitrary quadratic Jordan algebras.)

Sublemma. If $\mathfrak{B}$ is an outer ideal in $\mathfrak{A}$ with $\mathfrak{B}=\mathfrak{B}_{1}+\mathfrak{B}_{1 / 2}+\mathfrak{B}_{0}$ relative to an

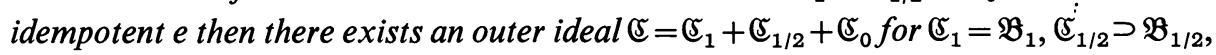
$\mathfrak{C}_{0}=\mathfrak{B}_{0}$ such that

(i) $P_{1 / 2}\left(\mathfrak{U}_{1 / 2} \mathfrak{E}_{1 / 2}\right) \subset \mathfrak{夭}_{1 / 2}$,

(ii) $P_{i}\left(\mathfrak{A}_{1 / 2} \circ \mathfrak{C}_{1 / 2}\right)=P_{i}\left(\mathfrak{A}_{1 / 2} \circ \mathfrak{B}_{1 / 2}\right)(i=1,0)$.

Proof. Set $\mathfrak{C}_{i}=\mathfrak{B}_{i}$, for $i=1,0$, and $\mathfrak{C}_{1 / 2}=\sum_{0}^{\infty} \mathfrak{B}_{1 / 2, n}$ where $\mathfrak{B}_{1 / 2,0}=\mathfrak{B}_{1 / 2}, \mathfrak{B}_{1 / 2, n+1}$ $=P_{1 / 2}\left(\mathfrak{A}_{1 / 2} \mathfrak{B}_{1 / 2, n}\right)$. Clearly $\mathfrak{C}_{1 / 2} \supset \mathfrak{B}_{1 / 2}$ satisfies (i) by construction, and to establish (ii) it is enough if $P_{i}\left(\mathfrak{A}_{1 / 2} \circ \mathfrak{B}_{1 / 2, n}\right) \subset P_{i}\left(\mathfrak{A}_{1 / 2} \circ \mathfrak{B}_{1 / 2}\right)$ for all $n$. This is trivial for $n=0$, and if true for $n$ then

$$
\begin{aligned}
P_{i}\left(\mathfrak{A}_{1 / 2} \circ \mathfrak{B}_{1 / 2, n+1}\right) & =P_{i}\left(\mathfrak{A}_{1 / 2} \circ P_{1 / 2}\left(\mathfrak{A}_{1 / 2} \mathfrak{B}_{1 / 2, n}\right)\right) \\
& =P_{i}\left(\mathfrak{B}_{1 / 2, n} \circ P_{1 / 2}\left(\mathfrak{A}_{1 / 2} \mathfrak{A}_{1 / 2}\right)\right) \quad(\text { by }(18)) \\
& \subset P_{i}\left(\mathfrak{B}_{1 / 2, n} \circ \mathfrak{A}_{1 / 2}\right) \subset P_{i}\left(\mathfrak{A}_{1 / 2} \circ \mathfrak{B}_{1 / 2}\right)
\end{aligned}
$$

by the induction hypothesis.

It remains to verify that $\mathfrak{E}$ is still outer. We have $U_{\mathfrak{A}} \mathfrak{C} \subset U_{\mathfrak{A}} \mathfrak{B}+U_{\mathfrak{A}} \mathbb{E}_{1 / 2} \subset \mathfrak{B}+U_{\mathfrak{A}} \mathfrak{E}_{1 / 2}$ so we need only show $U_{\mathfrak{A}} \mathbb{E}_{1 / 2} \subset \mathfrak{C}_{1 / 2}$. We have $U_{\mathfrak{A}_{0}} \mathbb{E}_{1 / 2}=U_{\mathfrak{A}_{1}} \mathbb{E}_{1 / 2}=0, U_{\mathfrak{A}_{0}, \mathfrak{H}_{1}} \mathbb{E}_{1 / 2}$ $=\mathfrak{A}_{0} \circ\left(\mathfrak{U}_{1} \circ \mathfrak{E}_{1 / 2}\right), U_{\mathfrak{A}_{1 / 2}, \mathfrak{A}_{i}} \mathfrak{E}_{1 / 2}=\left\{\mathfrak{U}_{1 / 2} \mathfrak{E}_{1 / 2} \mathfrak{A}_{i}\right\}=P_{i}\left\{\mathfrak{U}_{1 / 2} \circ\left(\mathfrak{E}_{1 / 2} \circ \mathfrak{A}_{i}\right)\right\}$, and $U_{\mathfrak{A}_{1 / 2}} \mathfrak{E}_{1 / 2}$ $=\mathfrak{U}_{1 / 2} \circ P_{i}\left(\mathfrak{U}_{1 / 2} \circ \mathfrak{C}_{1 / 2}\right)-\mathfrak{C}_{1 / 2} \circ P_{j}\left(\mathfrak{U}_{1 / 2}^{2}\right)$ so that all we need are

$$
\mathfrak{A}_{i} \circ \mathfrak{E}_{1 / 2} \subset \mathfrak{E}_{1 / 2}, \quad P_{i}\left\{\mathfrak{U}_{1 / 2} \circ \mathfrak{E}_{1 / 2}\right\}=P_{i}\left\{\mathfrak{A}_{1 / 2} \mathfrak{B}_{1 / 2}\right\} \subset \mathfrak{B}_{i},
$$

for $i=1,0$. In fact, we need only establish these for $\mathfrak{B}_{1 / 2, n}$ in place of $\mathfrak{夭}_{1 / 2}$. For $n=0$ these are clear since $\mathfrak{B}$ is outer, while by induction $\mathfrak{A}_{\mathfrak{i}} \circ \mathfrak{B}_{1 / 2, n+1}=\mathfrak{A}_{i} \circ P_{1 / 2}\left(\mathfrak{A}_{1 / 2} \mathfrak{B}_{1 / 2, n}\right)$ $=P_{1 / 2}\left\{\left(\mathfrak{A}_{i} \circ \mathfrak{A}_{1 / 2}\right) \mathfrak{B}_{1 / 2, n}\right\}($ by $(17)) \subset P_{1 / 2}\left(\mathfrak{U}_{1 / 2} \mathfrak{B}_{1 / 2, n}\right)=\mathfrak{B}_{1 / 2, n+1}$ and $P_{i}\left(\mathfrak{A}_{1 / 2} \circ \mathfrak{B}_{1 / 2, n+1}\right)$ 
$=P_{i}\left(\mathfrak{A}_{1 / 2} \circ P_{1 / 2}\left(\mathfrak{A}_{1 / 2} \mathfrak{B}_{1 / 2, n}\right)\right)=P_{i}\left(\mathfrak{B}_{1 / 2, n} \circ P_{1 / 2}\left(\mathfrak{A}_{1 / 2} \mathfrak{A}_{1 / 2}\right)\right)($ by $(18)) \subset P_{i}\left(\mathfrak{B}_{1 / 2, n} \circ \mathfrak{A}_{1 / 2}\right)$ $\subset P_{\mathfrak{i}}\left(\mathfrak{U}_{1 / 2} \circ \mathfrak{B}_{1 / 2}\right)$.

In the next lemma we will need to know that an outer ideal $\mathfrak{B}$ can be enlarged slightly so that $e \mathfrak{B} \subset \mathfrak{B}$. More generally,

Sublemma. If $\mathfrak{B}$ is an outer ideal in $\mathfrak{A}$ so is $\mathfrak{E}=\sum_{0}^{\infty} L_{x}^{n} \mathfrak{B}$ for any $x$, and $x \mathfrak{C} \subset \mathfrak{e}$.

Proof. Clearly $x \mathfrak{C} \subset \mathbb{C}$ by construction. To show $\mathbb{E}$ is outer, it suffices to show

$$
U_{a} L_{x}^{n} \mathfrak{B} \subset \sum_{m=0}^{n} L_{x}^{m} \mathfrak{B}
$$

for all $n$ and all $a \in \mathfrak{A}^{\prime}$. This is true for $n=0$ since $\mathfrak{B}$ is outer, $U_{a} \mathfrak{B} \subset \mathfrak{B}$; assuming the result for $n, U_{a} L_{x}^{n+1} \mathfrak{B}=\left\{U_{a} L_{x}\right\} L_{x}^{n} \mathfrak{B}=\left\{U_{a x, a}-R_{x} U_{a}\right\} L_{x}^{n} \mathfrak{B}$ (by (8)) $=\left\{U_{a x, a}-V_{x} U_{a}+L_{x} U_{a}\right\} L_{x}^{n} \mathfrak{B} \subset \sum_{m=0}^{n} L_{x}^{m} \mathfrak{B}+L_{x} \sum_{m=0}^{n} L_{x}^{m} \mathfrak{B}$ (by induction, noting $\left.V_{x}=U_{x, 1}\right) \subset \sum_{m=0}^{n+1} L_{x}^{m} \mathfrak{B}$. This completes the induction.

COROLlaRY. If $\mathfrak{B}$ is an outer ideal in $\mathfrak{A}, \mathfrak{B}=\mathfrak{B}_{1}+\mathfrak{B}_{1 / 2}+\mathfrak{B}_{0}$ relative to an idempotent $e$, then there exists an outer ideal $\mathfrak{E}=\mathfrak{E}_{1}+\mathfrak{E}_{1 / 2}+\mathfrak{C}_{0}$ with $\mathfrak{E}_{1}=\mathfrak{B}_{1}, \mathfrak{E}_{1 / 2} \supset \mathfrak{B}_{1 / 2}$, $\mathfrak{\mathfrak { V }}_{0}=\mathfrak{B}_{0}$ such that $e \mathfrak{C} \subset \mathfrak{C}$.

Proof. $\mathfrak{C}=\sum_{0}^{\infty} L_{e}^{n} \mathfrak{B}=\mathfrak{B}_{1}+\sum_{0}^{\infty} L_{e}^{n} \mathfrak{B}_{1 / 2}+\mathfrak{B}_{0}$.

Now we are ready to construct an ideal out of an outer ideal $\mathfrak{B}$; we shrink the components $\mathfrak{B}_{1}$ and $\mathfrak{B}_{0}$ but expand the component $\mathfrak{B}_{1 / 2}$.

LEMMA 4. If $\mathfrak{B}$ is an outer ideal in $\mathfrak{A}, \mathfrak{B}=\mathfrak{B}_{1}+\mathfrak{B}_{1 / 2}+\mathfrak{B}_{0}$ relative to $e$, then there is an ideal $\mathfrak{D}=\mathfrak{D}_{1}+\mathfrak{D}_{1 / 2}+\mathfrak{D}_{0}$ in $\mathfrak{A}$ with

$$
\mathfrak{B}_{1} \supset \mathfrak{D}_{1}, \quad \mathfrak{B}_{1 / 2} \subset \mathfrak{D}_{1 / 2}, \quad \mathfrak{B}_{0} \supset \mathfrak{D}_{0} .
$$

Proof. Given any outer ideal $\mathfrak{B}$ let $E(\mathfrak{B}) \supset \mathfrak{B}$ be the outer ideal invariant under $L_{e}$ constructed in the above corollary, $F(\mathfrak{B}) \supset \mathfrak{B}$ the outer ideal with $P_{1 / 2}\left(\mathfrak{H}_{1 / 2} \mathfrak{\mho}_{1 / 2}\right)$ $\subset \mathfrak{F}_{1 / 2}$ constructed in the first sublemma. We set $\mathfrak{B}_{0}=\mathfrak{B}, \mathfrak{B}_{2 n+1}=E\left(\mathfrak{B}_{2 n}\right), \mathfrak{B}_{2 n+2}$ $=F\left(\mathfrak{B}_{2 n+1}\right)$. Then the $\mathfrak{B}_{n}$ form an increasing sequence of outer ideals, and $\mathfrak{C}=\sum_{0}^{\infty} \mathfrak{B}_{n}$ is an outer ideal containing $\mathfrak{B}$. Since at each stage $\mathfrak{B}_{n}=\mathfrak{B}_{1}+\mathfrak{B}_{1 / 2, n}+\mathfrak{B}_{0}$ (the constructions $E$ and $F$ only increase the $\frac{1}{2}$-component) we see $\mathfrak{C}=\mathfrak{C}_{1}+\mathfrak{C}_{1 / 2}+\mathfrak{C}_{0}$ for $\mathfrak{S}_{1}=\mathfrak{B}_{1}, \mathfrak{C}_{1 / 2} \supset \mathfrak{B}_{1 / 2}, \mathfrak{C}_{0}=\mathfrak{B}_{0}$. We have $E(\mathfrak{C}) \subset \mathfrak{C}, F(\mathfrak{C}) \subset \mathfrak{C}$ since $E\left(\mathfrak{B}_{2 n}\right)=\mathfrak{B}_{2 n+1}$, $E\left(\mathfrak{B}_{2 n+1}\right)=\mathfrak{B}_{2 n+1}, F\left(\mathfrak{B}_{2 n+1}\right)=\mathfrak{B}_{2 n+2}, F\left(\mathfrak{B}_{2 n+2}\right)=\mathfrak{B}_{2 n+2}$. Thus $e \mathfrak{C} \subset \mathfrak{C}, P_{1 / 2}\left(\mathfrak{A}_{1 / 2} \mathfrak{C}_{1 / 2}\right)$ $\subset \mathfrak{C}_{1 / 2}$, and (since $\mathfrak{E}$ is outer) both $\mathfrak{U}_{i} \circ \mathfrak{C}_{1 / 2} \subset \mathfrak{C}_{1 / 2}$ and $\mathfrak{U}_{1 / 2} \circ P_{i}\left(\mathfrak{U}_{1 / 2} \circ \mathfrak{C}_{1 / 2}\right) \subset \mathfrak{U}_{1 / 2} \circ \mathfrak{C}_{i}$ $\subset \mathfrak{C}_{1 / 2}$ hold. By Proposition 1 we see $\mathscr{D}=\mathfrak{D}_{1}+\mathfrak{D}_{1 / 2}+\mathfrak{D}_{0}$ is an ideal for $\mathfrak{D}_{i}=$ $P_{i}\left(\mathfrak{U}_{1 / 2} \circ \mathfrak{S}_{1 / 2}\right) \subset \mathfrak{C}_{i}=\mathfrak{B}_{i}$ and $\mathfrak{D}_{1 / 2}=\mathfrak{E}_{1 / 2} \supset \mathfrak{B}_{1 / 2}$.

We can draw certain conclusions about simple algebras from these constructions. The major result guarantees that if $\mathfrak{A}$ is well behaved and contains an idempotent then $\mathfrak{A}^{+}$is well behaved.

THEOREM 1. A simple noncommutative Jordan algebra $\mathfrak{A}$ with idempotent $e \neq 0,1$ has no proper outer ideals. In particular, $\mathfrak{A}^{+}$is simple. 
Proof. The last remark follows since a proper ideal in $\mathfrak{A}^{+}$is a proper outer (and inner) ideal in $\mathfrak{A}$.

Suppose $\mathfrak{B} \neq \mathfrak{A}$ is outer, $\mathfrak{B}=\mathfrak{B}_{1}+\mathfrak{B}_{1 / 2}+\mathfrak{B}_{0}$ its Peirce decomposition relative to e. By Lemma 4 there exists an ideal $\mathfrak{D}=\mathfrak{D}_{1}+\mathfrak{D}_{1 / 2}+\mathfrak{D}_{0}$ in $\mathfrak{A}$ with $\mathfrak{D}_{i} \subset \mathfrak{B}_{i}$ and $\mathfrak{D}_{1 / 2} \supset \mathfrak{B}_{1 / 2}$. Now $\mathfrak{B}_{1}+\mathfrak{B}_{0}<\mathfrak{A}_{1}+\mathfrak{A}_{0}$ or else $e \in \mathfrak{B}_{1}$ implies $\mathfrak{A}_{1 / 2}=e \circ \mathfrak{A}_{1 / 2} \subset \mathfrak{B}$ and $\mathfrak{B}=\mathfrak{A}$, contradicting our choice of $\mathfrak{B}$. Thus $\mathfrak{D}_{1}+\mathfrak{D}_{0}<\mathfrak{A}_{1}+\mathfrak{A}_{0}$ too, so $\mathfrak{D} \neq \mathfrak{A}$, and by simplicity $\mathfrak{D}=0$. But then $\mathfrak{B}_{1 / 2} \subset \mathfrak{D}_{1 / 2}=0$, consequently $\mathfrak{B}_{i} \circ \mathfrak{A}_{1 / 2} \subset \mathfrak{B}_{1 / 2}=0$ implies $\mathfrak{B}_{i} \subset \mathbb{B}_{i}$ in the notation of Lemma 3 . We saw in that lemma that the $B_{i}$ are ideals in $\mathfrak{A}$, and ${B_{1}}_{1} \subset \mathfrak{A}_{1}<\mathfrak{A}$ if $e \neq 1$, while ${B_{0}}_{0} \subset \mathfrak{A}_{0}<\mathfrak{A}$ if $e \neq 0$, so again simplicity forces $\mathfrak{B}_{i}=0$. Then $\mathfrak{B}_{i}=0$ and $\mathfrak{B}=0$. Thus $\mathfrak{B}=0$ and $\mathfrak{B}=\mathfrak{A}$ are the only outer ideals.

Note that no finiteness restrictions were imposed on $\mathfrak{A}$. We next derive a similar theorem about simplicity of $\mathfrak{A}^{+}$in which we make no assumptions about idempotents, but instead place a finiteness and nondegeneracy condition on the algebra. In any quadratic Jordan algebra $\mathfrak{\Im}$ we define the derived series by $\mathfrak{\Im}^{(1)}=\mathfrak{J}, \mathfrak{\Im}^{(n+1)}$ $=U_{\mathfrak{J}^{(n)}} \mathfrak{\Im}^{(n)}$. $\mathfrak{I}$ is solvable if $\mathfrak{\Im}^{(n)}=0$ for some $n$. If $\mathfrak{B}$ is an ideal so are all $\mathfrak{B}^{(n)}$ (generally, if $\mathfrak{B}$ and $\mathfrak{C}$ are ideals so is $U_{\mathfrak{B}} \mathfrak{C}$ ).

LEMMA 5. If $\mathfrak{B}, \mathfrak{C}, \mathfrak{D}$ are ideals in a noncommutative Jordan algebra $\mathfrak{A}$ then $U_{\mathfrak{B}} \mathfrak{E}$ and $\{\mathfrak{B} \mathfrak{C} \mathfrak{D}\}$ are also ideals. If $\mathfrak{B}$ is an ideal in $\mathfrak{A}^{+}$then $\mathfrak{A}_{\mathfrak{B}^{(n)}}+\mathfrak{B}^{(n)} \mathfrak{A} \subset \mathfrak{B}^{(n-1)}$, so that $\bigcap_{n} \mathfrak{B}^{(n)}$ is an ideal in $\mathfrak{A}$.

Proof. $U_{\mathfrak{B}} \mathfrak{E}$ is a left ideal since by (8) $L_{x} U_{\mathfrak{B}} \mathfrak{E}=-U_{\mathfrak{B}} R_{x} \mathfrak{E}+U_{\mathfrak{B}, x \mathfrak{B}} \mathfrak{E} \subset U_{\mathfrak{B}} \mathfrak{E}$ because $\mathfrak{B}$ is a left and $\mathfrak{E}$ a right ideal, and similarly $U_{\mathfrak{B}} \mathfrak{E}$ is a right ideal. The same argument works for $\{\mathfrak{B} \mathfrak{C} \mathfrak{D}\}: L_{x}\{\mathfrak{B} \mathfrak{C} \mathfrak{D}\}=L_{x} U_{\mathfrak{B}, \mathfrak{D}} \mathfrak{E}=-U_{\mathfrak{B}, \mathfrak{D}} R_{x} \mathfrak{E}+U_{x \mathfrak{B}, \mathscr{D}} \mathfrak{E}+$ $U_{\mathfrak{B}, x \mathfrak{D}} \mathfrak{C} \subset\{\mathfrak{B} \mathbb{C} \mathfrak{D}\}$ since $\mathfrak{B}, \mathfrak{D}$ are left and $\mathfrak{C}$ right ideals.

If $\mathfrak{B}$ is only an ideal in $\mathfrak{A}^{+}$we use (8) and induction to obtain

$$
\begin{aligned}
& \mathfrak{Y} \mathfrak{B}^{(n+1)}=L_{\mathfrak{Y}} U_{\mathfrak{B}^{(n)}} \mathfrak{P}^{(n)}=-U_{\mathfrak{P}^{(n)}} R_{\mathfrak{A}} \mathfrak{P}^{(n)}+U_{\mathfrak{Y} \mathfrak{B}}(n) \mathfrak{Q}^{(n)} \mathfrak{B}^{(n)} \\
& \subset U_{\mathfrak{B}^{(n)}} \mathfrak{B}^{(n-1)}+U_{\mathfrak{B}^{(n-1)}, \mathfrak{Q}^{(n)} \mathfrak{B}^{(n)}} \\
& \subset U_{\mathfrak{B}^{(n-1)}} \mathfrak{B}^{(n-1)}=\mathfrak{B}^{(n)}
\end{aligned}
$$

for $n \geqq 1$ (for $n=0$ we agree $\mathfrak{B}^{(0)}=\mathfrak{Q}$ ). Similarly $\mathfrak{B}^{(n+1)} \mathfrak{Q} \subset \mathfrak{B}^{(n)}$, so $\mathfrak{E}=\bigcap \mathfrak{B}^{(n)}$ has $\mathfrak{A C}+\mathfrak{C} \mathfrak{A} \subset \bigcap\left(\mathfrak{A} \mathfrak{B}^{(n+1)}+\mathfrak{B}^{(n+1)} \mathfrak{A}\right) \subset \bigcap \mathfrak{B}^{(n)}=\mathfrak{C}$ so that $\mathfrak{E}$ is an ideal in $\mathfrak{A}$.

THEOREM 2. If $\mathfrak{A}$ is a simple noncommutative Jordan algebra such that $\mathfrak{A}^{+}$has the descending chain condition on ideals (in particular, if it has d.c.c. on inner ideals) but contains no solvable ideals, then $\mathfrak{X}^{+}$is simple.

Proof. If $\mathfrak{B}$ were a proper ideal in $\mathfrak{A}^{+}$then the $\mathfrak{B}^{(n)}$ would form a decreasing sequence of ideals, hence $\mathfrak{B}^{(N)}=\mathfrak{B}^{(N+1)}=\cdots$ from some point on by the d.c.c. Thus $\mathfrak{E}=\bigcap \mathfrak{B}^{(n)}=\mathfrak{B}^{(N)}$ is an ideal in $\mathfrak{A}$. It cannot be all of $\mathfrak{A}$ since it is contained in $\mathfrak{B}$, yet it cannot be zero because $\mathfrak{B} \neq 0$ is not solvable. But it cannot be a proper ideal in the simple algebra $\mathfrak{A}$ either, a contradiction.

We shall need one further result concerning Peirce spaces. An algebra is semiprime if it contains no (nonzero) trivial elements, an element $z$ being trivial if $U_{z}=0$. 
LeMma 6. If $\mathfrak{A}$.is semiprime then any $x_{1 / 2} \in \mathfrak{A}_{1 / 2}$ such that $P_{1}\left(x_{1 / 2} \circ \mathfrak{A}_{1 / 2}\right)=0$ or $x_{1 / 2} \circ \mathfrak{A}_{0}=0$ is zero. In particular, $\mathfrak{A}_{0}=0$ implies $e$ is the unit for $\mathfrak{A}, \mathfrak{A}=\mathfrak{A}_{1}$.

Proof. If $P_{1}\left(x_{1 / 2} \circ \mathfrak{A}_{1 / 2}\right)=0$ (or $x_{1 / 2} \circ \mathfrak{A}_{0}=0$ ), then $U_{x_{1 / 2}} \mathfrak{A}_{0}=P_{1}\left\{x_{1 / 2} \circ\left(x_{1 / 2} \circ \mathfrak{A}_{0}\right)\right\}$ $=0$ by (19) (or $\left.=P_{1}\left\{x_{1 / 2}\left(x_{1 / 2} \circ \mathfrak{A}_{0}\right)\right\}=0\right)$. Then any $z_{0} \in U_{x_{1 / 2}} \mathfrak{A}_{1}$ would have $U_{z_{0}} \mathfrak{A}$ $=U_{z_{0}} \mathfrak{A}_{0} \subset U_{x_{1 / 2}} U_{\mathfrak{A}_{1}} U_{x_{1 / 2}} \mathfrak{A}_{0}=0$, so $z_{0}$ would be trivial; by semiprimeness $z_{0}=0$ and we have $\dot{U}_{x_{1 / 2}} \mathfrak{A}_{1}=0$ too. Thus $P_{0}\left(x_{1 / 2}^{2}\right)=U_{x_{1 / 2}} e=0$, implying $x_{1 / 2}^{2}=z_{1} \in \mathfrak{A}_{1}$. A similar argument shows $U_{z_{1}} \mathfrak{A}=U_{z_{1}} \mathfrak{A}_{1}=U_{x_{1 / 2}} U_{x_{1 / 2}} \mathfrak{A}_{1}=0$ so $z_{1}=0$ and $x_{1 / 2}^{2}=0$. Then $U_{x_{1 / 2}} \mathfrak{H}_{1 / 2}=x_{1 / 2} \circ P_{1}\left(x_{1 / 2} \circ \mathfrak{A}_{1 / 2}\right)-\mathfrak{A}_{1 / 2} \circ P_{0}\left(x_{1 / 2}^{2}\right)=0$ (or $=x_{1 / 2} \circ P_{0}\left(x_{1 / 2} \circ \mathfrak{A}_{1 / 2}\right)$ $\left.-\mathfrak{A}_{1 / 2} \circ P_{1}\left(x_{1 / 2}^{2}\right)=0\right)$. This shows that $U_{x_{1 / 2}} \mathfrak{A}=0$ and $x_{1 / 2}=0$.

4. Interconnected idempotents. Two orthogonal idempotents $e_{1}, e_{2}$ in a quadratic Jordan algebra $\mathfrak{\Im}$ are interconnected if $U_{\mathfrak{I}_{12}} \mathfrak{\Im}_{11}=\mathfrak{\Im}_{22}$ and $U_{\mathfrak{I}_{12}} \mathfrak{\Im}_{22}=\mathfrak{\Im}_{11}$. More generally, a Peirce decomposition $\mathfrak{\Im}=\sum_{0 \leqq i, j \leqq n-1} \Im_{i j}$ relative to orthogonal idempotents $e_{1}, \ldots, e_{n-1}$ is interconnected if

(I') $U_{\mathfrak{I}_{i},} \Im_{j j}=\Im_{i i}(i \neq j)$,

(II') $x_{i 0} \circ \Im_{00}=0 \Rightarrow x_{i 0}=0(i \neq 0)$,

(III') $x_{00} \circ \mathfrak{\Im}_{i 0}=0 \Rightarrow x_{00}=0(i \neq 0)$.

The latter are nondegeneracy conditions: if $e_{0}$, exists (i.e. if $\mathfrak{\Im}$ is unital), (II') is automatic, and (III') holds if $\mathfrak{\Im}$ has no extreme radical (so always when $\frac{1}{2} \in \Phi$ ). In the particular case where $\mathfrak{\Im}=\mathfrak{U}^{+}$for a noncommutative Jordan algebra $\mathfrak{A}$ we see $\mathfrak{A}_{i i}$ is spanned by the $U_{x_{i j}} y_{j j}=P_{i i}\left(x_{i j} \circ x_{i j} y_{j j}\right)$ (by (19)) $\subset P_{i i}\left(\mathfrak{A}_{i j} \circ \mathfrak{A}_{i j}\right)$, and also $\mathfrak{A}_{0 i} \subset \mathfrak{A}_{0 i} \circ \mathfrak{A}_{i i} \quad$ (since $e_{i}$ exists for $\left.i \neq 0\right)=\mathfrak{A}_{0 i} \circ U_{\mathfrak{A}_{i 0}} \mathfrak{A}_{00}=-\mathfrak{A}_{00} \circ U_{\mathfrak{A}_{i 0}} \mathfrak{A}_{0 i}$ $+\left\{\mathfrak{U}_{0 i} \mathfrak{A}_{i 0} \mathfrak{A}_{00}\right\} \circ \mathfrak{A}_{i 0}$ (linearizing (6)) $\subset \mathfrak{A}_{00} \circ \mathfrak{A}_{i 0}$. This leads us to say that a Peirce decomposition $\mathfrak{U}=\sum_{0 \leqq i, j \leqq n-1} \mathfrak{A}_{i j}$ (of length $n$ ) of a noncommutative Jordan algebra $\mathfrak{A}$ is interconnected if

$$
\begin{array}{rll}
\text { (I) } & P_{i i}\left\{\mathfrak{A}_{i j} \circ \mathfrak{A}_{i j}\right\}=\mathfrak{A}_{i i} & (i \neq j), \\
\text { (II) } & x_{i 0} \circ \mathfrak{A}_{00}=0 \Rightarrow x_{i 0}=0 & (i \neq 0), \\
\text { (III) } & x_{00} \circ \mathfrak{A}_{i 0}=0 \Rightarrow x_{00}=0 & (i \neq 0), \\
\text { (IV) } & \mathfrak{A}_{i 0} \circ \mathfrak{A}_{00}=\mathfrak{A}_{i 0} & (i \neq 0) .
\end{array}
$$

We have seen that a Peirce decomposition which is interconnected in $\mathfrak{A}^{+}$is interconnected in $\mathfrak{A}$. The converse is also true. If a decomposition is interconnected in $\mathfrak{A}$ we establish (I') by $U_{\mathfrak{A}_{i j}} \mathfrak{A}_{j j}=P_{i i}\left\{\mathfrak{A}_{i j}\left(\mathfrak{A}_{i j} \circ \mathfrak{A}_{j j}\right)\right\}$ (by (19)) $=P_{i i}\left(\mathfrak{A}_{i j} \mathfrak{A}_{i j}\right)$ (using (IV) if $j=0)=P_{i i}\left(\mathfrak{A}_{i j} \circ \mathfrak{A}_{i j}\right)\left(\right.$ by $\left.(15)^{\prime}\right)=\mathfrak{A}_{i i}$ (by (I)).

Note once more than (II) and (IV) are superfluous if $e_{0}$ exists, i.e. $\mathfrak{A}$ is unital. Moreover, (IV) is also superfluous if $n \geqq 3$; this will follow from the general result that (I) implies

(I') $\mathfrak{A}_{i j} \circ \mathfrak{A}_{j k}=\mathfrak{A}_{i k}(i, j, k \neq)$

since then $\mathfrak{A}_{i 0} \circ \mathfrak{A}_{00}=\mathfrak{A}_{i 0} \circ \mathfrak{H}_{j 0}^{2}$ (by (I), where $j \neq i, 0$ exists if $\left.n \geqq 3\right)=\left(\mathfrak{A}_{i 0} \circ \mathfrak{A}_{j 0}\right) \circ \mathfrak{A}_{j 0}$ (by $\left.(20)^{\prime}\right)=\mathfrak{A}_{i j} \circ \mathfrak{A}_{j 0}$ (if $\left(\mathrm{I}^{\prime}\right)$ holds) $=\mathfrak{A}_{i 0}$ (by $\left(\mathrm{I}^{\prime}\right)$ again). To establish $\left(\mathrm{I}^{\prime}\right)$, we may assume $k \neq 0$ (a similar argument applies if $i \neq 0$ ) so that $\mathfrak{A}_{i k} \subset \mathfrak{A}_{i k} \circ \mathfrak{A}_{k k}=\mathfrak{A}_{i k} \circ \mathfrak{A}_{j k}^{2}$ (by (I)) $=\left(\mathfrak{A}_{i k} \circ \mathfrak{A}_{j k}\right) \circ \mathfrak{A}_{j k}\left(\right.$ by $\left.(20)^{\prime}\right) \subset \mathfrak{A}_{i j} \circ \mathfrak{A}_{j k}$. 
It is more work to show that (III) is superfluous in the unital case. We claim more, namely

$$
x_{i i} \circ \mathfrak{U}_{i j}=0 \Rightarrow x_{i i} \mathfrak{U}=\mathfrak{U} x_{i i}=0 .
$$

We have $x_{i i} \mathfrak{A}_{i j}^{2}=\mathfrak{A}_{i j}^{2} x_{i i}=0$ by (20), so by (I), $x_{i i} \mathfrak{A}_{i i}=\mathfrak{A}_{i i} x_{i i}=0$. By (14) we have $x_{i i} \mathfrak{A}_{i j}=\mathfrak{A}_{i j} x_{i i}=0$. For $k \neq i, j$, we have $x_{i i} \circ \mathfrak{A}_{i k}=x_{i i} \circ\left(\mathfrak{A}_{i j} \circ \mathfrak{A}_{j k}\right)$ (by $\left.\left(\mathrm{I}^{\prime}\right)\right)$ $=\left(x_{i i} \circ \mathfrak{A}_{i j}\right) \circ \mathfrak{A}_{j k}$ (by (16)) $=0$, so by (14) again $x_{i i} \mathfrak{A}_{i k}=\mathfrak{A}_{i k} x_{i i}=0$. By Peirce orthogonality these are enough to show that $x_{i i}$ annihilates $\mathfrak{A}$. If $e_{i i}$ exists, this gives $x_{i i}=0$. More generally

(III') $x_{i j} \circ \mathfrak{A}_{j k}=0 \Rightarrow x_{i j}=0(k \neq i, j)$.

We have just seen this for $i=j \neq 0$, while for $i=j=0$ it follows from (III). If $i \neq j$ note $x_{i j} \mathfrak{A}_{j k}^{2}=\mathfrak{A}_{j k}^{2} x_{i j}=0$ by (20)', so by (I) $x_{i j} \mathfrak{A}_{j j}=\mathfrak{A}_{j j} x_{i j}=0$; if $e_{j}$ exists this implies $x_{i j}=0$ directly, otherwise for $j=0$ we use (II).

In the presence of interconnectivity we can improve on the Peirce relation (GPR 4).

(V) $\mathfrak{A}_{i j}^{2} \subset \mathfrak{A}_{i i}+\mathfrak{A}_{j j}$ if $n \geqq 3$.

This is a consequence of $\left(\mathrm{III}^{\prime}\right)$ because $\mathfrak{A}_{j k} \circ P_{i j}\left(\mathfrak{H}_{i j}^{2}\right)=P_{i j}\left\{\left(\mathfrak{A}_{j k} \circ \mathfrak{A}_{i j}\right) \mathfrak{A}_{i j}\right\}$ (by (17)) $\subset P_{i j}\left(\mathfrak{U}_{i k} \mathfrak{A}_{i j}\right) \subset P_{i j}\left(\mathfrak{U}_{j k}\right)=0$.

It is also useful to know that when $e_{i}$ exists, (I) is equivalent to

(I*) $e_{i} \in P_{i i}\left(\mathfrak{A}_{i j} \circ \mathfrak{A}_{i j}\right)$

because, by Proposition $1, P_{i i}\left(\mathfrak{A}_{i j} \circ \mathfrak{A}_{i j}\right)$ it an ideal in $\mathfrak{A}_{i i}$, and therefore if it contains $e_{i}$ it contains all of $\mathfrak{A}_{i i}$.

We remark that an interconnected algebra remains interconnected under any free scalar extension $\Omega$ of $\Phi$ (in particular, if $\Omega$ is an extension of a field $\Phi$ ). Indeed, conditions (I) and (IV) are inherited by $\mathfrak{A}_{\Omega}$ for an arbitrary extension $\Omega$, and if $\Omega$ is free over $\Phi$ with basis $\left\{\omega_{\alpha}\right\}$ then $\mathfrak{A}_{\Omega}=\oplus \omega_{\alpha} \mathfrak{A}$ inherits (II), (III) also: if $x_{i 0}$ $=\sum \omega_{\alpha} x_{i 0}^{\alpha}\left(x_{i 0}^{\alpha} \in \mathfrak{A}\right)$ has $x_{i 0} \circ \mathfrak{A}_{00}=0$ then $\sum \omega_{\alpha}\left(x_{i 0}^{\alpha} \circ \mathfrak{A}_{00}\right)=0$ implies $x_{i 0}^{\alpha} \circ \mathfrak{A}_{00}=0$ for all $\alpha$, hence by (II) in $\mathfrak{A}$ all $x_{i 0}^{\alpha}=0$ and $x_{i 0}=0$. (Similarly for (III).)

Further, if $\mathfrak{A}$ is a unital interconnected algebra and $\mathfrak{M}$ a unital bimodule (in the sense of [3, p. 191]) then the split null extension $\mathfrak{E}=\mathfrak{A} \oplus \mathfrak{M}$ is also interconnected. More generally, if $\mathfrak{E}$ is any unital algebra containing an interconnected algebra $\mathfrak{A}$ as a unital subalgebra then $\leftleftarrows$ inherits interconnectivity from $\mathfrak{A}$. ((II), (III), (IV) are automatic and (I) equivalent to (I*) in the unital case, and $\mathbb{E}$ inherits (I*) since $e_{i} \in P_{i i}\left(\mathfrak{A}_{i j} \circ \mathfrak{A}_{i j}\right)$ implies $e_{i} \in P_{i i}\left(\mathfrak{F}_{i j} \circ \mathfrak{F}_{i j}\right)$.)

As with the Jordan case, interconnectivity is preserved if certain of the idempotents are "lumped together": if $e_{1}, \ldots, e_{n-1}$ afford an interconnected decomposition so do $e_{1}^{\prime}, \ldots, e_{m-1}^{\prime}$ where $e_{j}^{\prime}=\sum_{i \in I(j)} e_{i}$ for any decomposition $I=\{1,2, \ldots, n-1\}=I(1) \cup \cdots \cup I(m-1)$ into disjoint sets. Indeed, (II), (III), (IV) are clear since $\mathfrak{A}_{00}^{\prime}=\mathfrak{A}_{00}, \mathfrak{A}_{0 j}^{\prime}=\sum_{i \in I(j)} \mathfrak{A}_{0 i}$, while for (I) we observe

$$
\begin{aligned}
P_{j j}\left(\mathfrak{H}_{j k}^{\prime} \circ \mathfrak{A}_{j k}^{\prime}\right) & =\sum_{r, s \in I(j), t \in I(k)} P_{r s}\left(\mathfrak{A}_{r t} \circ \mathfrak{A}_{s t}\right)=\sum_{r} P_{r r}\left(\mathfrak{A}_{r t} \circ \mathfrak{A}_{r t}\right)+\sum_{r \neq s} P_{r s}\left(\mathfrak{A}_{r t} \circ \mathfrak{A}_{s t}\right) \\
& =\sum \mathfrak{A}_{r r}+\sum \mathfrak{A}_{r s} \quad(\text { by }(\mathrm{I}),(\mathrm{V})) \\
& =\mathfrak{A}_{j j .}^{\prime} .
\end{aligned}
$$


The importance of interconnectivity derives from the

TheOREM 3. Any Peirce decomposition of an arbitrary simple noncommutative Jordan algebra is interconnected.

Proof. Let $\mathfrak{A}=\sum_{0 \leqq i, j \leqq n-1} \mathfrak{A}_{i j}$ be a Peirce decomposition relative to $e_{1}, \ldots, e_{n-1}$. We may assume $n \geqq 2$ or else $\mathfrak{A}=\mathfrak{A}_{00}$ is vacuously interconnected.

We first tackle (I). By Lemma 1 we know $\mathfrak{B}=\mathfrak{B}_{1}+\mathfrak{B}_{1 / 2}+\mathfrak{B}_{0}=P_{1}\left(\mathfrak{U}_{1 / 2}^{2}\right)+\mathfrak{A}_{1 / 2}$ $+P_{0}\left(\mathfrak{U}_{1 / 2}^{2}\right)$ is an ideal for any idempotent $e$; if $e \neq 1,0$ then $\mathfrak{B} \neq 0$ since otherwise $\mathfrak{A}_{1 / 2}=0, \mathfrak{A}=\mathfrak{A}_{1} \oplus \mathfrak{A}_{0}$, and by simplicity $\mathfrak{A}=\mathfrak{A}_{1}$ or $\mathfrak{A}=\mathfrak{A}_{0}$, consequently $e=1$ or $e=0$. Thus $\mathfrak{B}=\mathfrak{A}$ for any $e \neq 1,0$. Taking $e=e_{j}(j \neq 0)$ we have $\mathfrak{A}_{1 / 2}=\sum_{i \neq j} \mathfrak{A}_{i j}$ so $\mathfrak{A}_{i i}=P_{i i}(\mathfrak{B})=P_{i i}\left(\mathfrak{B}_{0}\right)=P_{i i}\left(\mathfrak{U}_{1 / 2}^{2}\right)=P_{i i}\left(\mathfrak{H}_{i j} \circ \mathfrak{A}_{i j}\right)$ by the Peirce relations. Similarly if $j=0$ we take $e=e_{1}+\cdots+e_{n-1}$ so $\mathfrak{A}_{1 / 2}=\sum \mathfrak{A}_{i 0}$ and $\mathfrak{A}_{i i}=P_{i i}(\mathfrak{B})=P_{i i}\left(\mathfrak{B}_{1}\right)=P_{i i}\left(\mathfrak{U}_{1 / 2}^{2}\right)$ $=P_{i i}\left(\mathfrak{A}_{i 0} \circ \mathfrak{A}_{i 0}\right)$. Thus $\mathfrak{A}_{i i}=P_{i i}\left(\mathfrak{A}_{i j} \circ \mathfrak{A}_{i j}\right)$ in all cases.

Next we attack (IV). By Theorem 1 we know $\mathfrak{A}$ has no proper outer ideals (always assuming $n \geqq 2)$; this guarantees $\operatorname{Rad} \mathfrak{X}^{+}=0\left(\operatorname{Rad} \mathfrak{A}^{+}\right.$is an outer ideal but contains no idempotents, so it cannot be all of $\mathfrak{A}$ ) and therefore $\mathfrak{A}$ contains no trivial elements [4, pp. 676-677]. By Lemma 6, this in turn guarantees $\mathfrak{A}_{0} \neq 0$. By Lemma 2, $\mathfrak{B}=\mathfrak{B}_{1}+\mathfrak{B}_{1 / 2}+\mathfrak{B}_{0}$ is an ideal for $\mathfrak{B}_{1 / 2}=\mathfrak{U}_{0} \circ \mathfrak{A}_{1 / 2}$. Now $\mathfrak{B}_{1 / 2} \neq 0$ or else $\mathfrak{A}_{0}=Z_{0}$ (in the notation of Lemma 3), whereas $e \notin \mathbf{Z}_{0}$ implies the ideal $\boldsymbol{Z}_{0}$ is not $\mathfrak{A}$ and hence by simplicity must be zero, leading to $\mathfrak{A}_{0}=0$ which we just saw was impossible. Thus $\mathfrak{B} \neq 0$; by simplicity $\mathfrak{B}=\mathfrak{A}$, hence $\mathfrak{A}_{0} \circ \mathfrak{A}_{1 / 2}=\mathfrak{B}_{1 / 2}=\mathfrak{U}_{1 / 2}$. Applying this to $e=e_{1}+\cdots+e_{n-1}$ we obtain $\sum \mathfrak{A}_{i 0}=\mathfrak{A}_{1 / 2}=\mathfrak{A}_{0} \circ \mathfrak{A}_{1 / 2}=\sum \mathfrak{A}_{00} \circ \mathfrak{A}_{0 i}$ so that $\mathfrak{A}_{i 0}=\mathfrak{A}_{00} \circ \mathfrak{A}_{i 0}$.

Next we turn to (II). If $x_{i 0} \circ \mathfrak{A}_{00}=0$ then $x_{i 0}$ is trivial by Lemma 6 , so $x_{i 0}=0$.

Finally, we come to (III). Now $x_{00} \circ \mathfrak{A}_{i 0}=0$ implies $x_{00} \circ \mathfrak{A}_{j 0}=x_{00} \circ\left(\mathfrak{A}_{i 0} \circ \mathfrak{A}_{i j}\right)$ (by $\left.\left(\mathrm{I}^{\prime}\right)\right)=\left(x_{00} \circ \mathfrak{A}_{i 0}\right) \circ \mathfrak{A}_{i j}$ (by (16)) $=0$ for any $j \neq i, 0$ so $x_{00} \in \mathfrak{Z}_{0}$ relative to $e=e_{1}+\cdots+e_{n-1}$ (as in Lemma 3): $x_{00} \circ \mathfrak{A}_{1 / 2}=0$. Since $\boldsymbol{B}_{0} \neq \mathfrak{A}$ is an ideal we must have $3_{0}=0$, therefore $x_{00}=0$.

Again note that this result is completely general, no finiteness condition being imposed.

We now come to some basic structural properties of interconnected algebras. We say $\mathfrak{A}$ is $n$-interconnected if it has some interconnected Peirce decomposition of length $n$. We will see that $n$-interconnected algebras for $n \geqq 3$ are well behaved.

THEOREM 4. If $\mathfrak{A}$ is $n$-interconnected for $n \geqq 3$ then there is an element $\gamma$ in the centroid $\Gamma$ such that

$$
e_{i} x_{i j} e_{i}=\gamma\left(x_{i j}\right) \quad\left(x_{i j} \in \mathfrak{A}_{i j}, i \neq j\right) .
$$

Proof. The first step is to define $\gamma$. If $\mathfrak{A}=\sum \mathfrak{A}_{i j}$ is the $n$-interconnected Peirce decomposition, we define a linear map on $\mathfrak{A}_{i j}$ for $i \neq j$ by

$$
T\left(x_{i j}\right)=e_{i} x_{i j} e_{i}=e_{j} x_{i j} e_{j}
$$

(recall $e_{i} x=x e_{j}$ ). We claim that for $i, j, k \neq$ (and $e_{0}$ being interpreted as an element of the unital extension $\mathfrak{A}^{\prime}$ ) 
(i) $T\left(x_{i j} y_{j k}\right)=T\left(x_{i j}\right) y_{j k}=x_{i j} T\left(y_{j k}\right)=\left(e_{i} x_{i j}\right)\left(e_{k} y_{j k}\right)$,

(ii) $T\left(x_{i i} y_{i j}\right)=x_{i i} T\left(y_{i j}\right)$.

For (i), $\quad T\left(x_{i j} y_{j k}\right)=L_{e_{i}} R_{e_{i}}\left(x_{i j} y_{j k}\right)=L_{e_{i}} R_{e_{i}} R_{y_{j k}}\left(x_{i j}\right)=R_{y_{j k}} L_{e_{i}} R_{e_{i}}\left(x_{i j}\right) \quad$ (by $=T\left(x_{i j}\right) y_{j k}$, similarly $T\left(x_{i j} y_{j k}\right)=L_{e_{k}} R_{e_{k}}\left(x_{i j} y_{j k}\right)=x_{i j} T\left(y_{j k}\right)$, and finally $T\left(x_{i j} y_{j k}\right)$ $=L_{e_{i}} L_{e_{k}}\left(x_{i j} y_{j k}\right)=L_{e_{i}} L_{x_{i j}} L_{e_{k}}\left(y_{j k}\right)=e_{i}\left\{x_{i j}\left(e_{k} y_{j k}\right)\right\}=\left(e_{i} x_{i j}\right)\left(e_{k} y_{j k}\right)$. The same trick works for (ii)', $T\left(x_{i i} y_{i j}\right)=L_{e,} R_{e j}\left(x_{i i} y_{i j}\right)=x_{i i} T\left(y_{i j}\right)$.

Our next step is to extend $T$ to the Peirce spaces $\mathfrak{A}_{\mathfrak{i i}}$. Here we use interconnectivity: $\mathfrak{A}_{i i}$ is spanned by the $P_{i i}\left(x_{i j}^{2}\right)$ for $i \neq j$, and we set

$$
T\left(P_{i i}\left(x_{i j}^{2}\right)\right)=P_{i i}\left\{\left(e_{i} x_{i j}\right)^{2}\right\} .
$$

We must first show this is well defined: for $i, j, k \neq$,

$$
P_{i i}\left\{\sum x_{i j}^{2}\right\}=P_{i i}\left\{\sum y_{i k}^{2}\right\} \Rightarrow P_{i i}\left\{\sum\left(e_{i} x_{i j}\right)^{2}\right\}=P_{i i}\left\{\sum\left(e_{i} y_{i k}\right)^{2}\right\}
$$

(in particular $\left.P_{i i}\left\{\sum x_{i j}^{2}\right\}=0 \Rightarrow P_{i i}\left\{\sum\left(e_{i} x_{i j}\right)^{2}\right\}=0\right)$. Now because of interconnectivity (III'), $a_{i i} \circ z_{i k}=b_{i i} \circ z_{i k}$ for all $z_{i k} \in \mathfrak{A}_{i k}$ implies $a_{i \mathfrak{i}}=b_{i i}$, so we want to show $P_{i i}\left\{\sum\left(e_{i} x_{i j}\right)^{2}\right\} \circ z_{i k}=P_{i i}\left\{\sum\left(e_{i} y_{i k}\right)^{2}\right\} \circ z_{i k}$ agree for all $z_{i k}$, and since $\mathfrak{A}_{i k}=\mathfrak{A}_{i j} \circ \mathfrak{A}_{j k}$ by $\left(\mathrm{I}^{\prime}\right)$ we need only consider $z_{i k}=w_{i j} \circ v_{j k}$. Here $a_{i i} \circ z_{i k}=P_{i i}\left\{\sum\left(e_{i} x_{i j}\right)^{2}\right\} \circ z_{i k}$ $=\sum\left(e_{i} x_{i j}\right)^{2} \circ z_{i k}=\sum\left(e_{i} x_{i j}\right) \circ\left\{\left(e_{i} x_{i j}\right) \circ z_{i k}\right\} \quad$ (by $\left.(20)^{\prime}\right)=\sum\left(e_{i} x_{i j}\right) \circ\left\{\left(x_{i j} e_{j}\right) \circ z_{i k}\right\}$ $=\sum\left(e_{i} x_{i j}\right) \circ\left\{\left(x_{i j} \circ z_{i k}\right) e_{j}\right\} \quad$ (by (13)) $=\sum T\left\{x_{i j} \circ\left(x_{i j} \circ z_{i k}\right)\right\}$ (by (i)) $=T\left(\sum x_{i j}^{2} \circ z_{i k}\right)$ $=T\left(z_{i k} \circ P_{i i}\left(\sum x_{i j}^{2}\right)\right) \quad$ and $\quad b_{i i} \circ z_{i k}=b_{i i} \circ\left(w_{i j} \circ v_{j k}\right)=\left(b_{i i} \circ w_{i j}\right) \circ v_{j k} \quad$ (by (16)) $=T\left(w_{i j} \circ P_{i \imath}\left(\sum y_{i k}^{2}\right)\right) \circ v_{j k}$ (by the same argument as before) $=T\left(\left\{w_{i j} \circ P_{i \mathfrak{i n}}\left(\sum y_{i k}^{2}\right)\right\} \circ v_{j k}\right)$ (by (i)) $=T\left(\left\{w_{i j} \circ v_{j k}\right\} \circ P_{i i}\left(\sum y_{i k}^{2}\right)\right)=T\left(z_{i k} \circ P_{i i}\left(\sum y_{i k}^{2}\right)\right)$.

We have actually shown $T\left(x_{i i} \circ z_{i k}\right)=T\left(x_{i i}\right) \circ z_{i k}$ for any $x_{i i}=P_{i i}\left(\sum x_{i j}^{2}\right)$ in $\mathfrak{A}_{i i}$; from this we obtain

(ii)" $T\left(x_{i i} y_{i j}\right)=T\left(x_{i i}\right) y_{i j}$ since $T\left(x_{i i}\right) y_{i j}=e_{i}\left\{T\left(x_{i i}\right) \circ y_{i j}\right\}=e_{i}\left\{T\left(x_{i i} \circ y_{i j}\right)\right\}=T\left(e_{i}\left(x_{i i} \circ y_{i j}\right)\right)$ (by (ii)', or since $L_{e_{i}}$ commutes with $\left.T=L_{e_{i}} R_{e_{i}}\right)=T\left(x_{i i} y_{i j}\right)$ using (14). Since $T$ is linear and preserves Peirce spaces, the only other formulas necessary in addition to (i)-(ii) to show $T$ belongs to the centroid $T(x y)=T(x) y=x T(y)$ are

(iii) $T\left(x_{i j} y_{i j}\right)=T\left(x_{i j}\right) y_{i j}=x_{i j} T\left(y_{i j}\right)$,

(iv) $T\left(x_{i i} y_{i i}\right)=T\left(x_{i i}\right) y_{i i}=x_{i i} T\left(y_{i i}\right)$.

For (iii), $T\left(P_{i i}\left\{z_{i j} \circ w_{i j}\right\}\right)=P_{i i}\left\{e_{i} z_{i j} \circ e_{i} w_{i j}\right\}$ (linearizing the definition of $T$ ) $=P_{i i}\left\{z_{i j} \circ e_{i} w_{i j} e_{i}\right\}$ (by (15)) $=P_{i i}\left\{z_{i j} \circ T\left(w_{i j}\right)\right\}$ so $T\left(P_{i i}\left\{x_{i j} y_{i j}\right\}\right)=T\left(P_{i i}\left\{e_{i} x_{i j} \circ y_{i j}\right\}\right)$ (by (15)) $=P_{i i}\left\{e_{i} x_{i j} \circ T\left(y_{i j}\right)\right\}$ (by the foregoing) $=P_{i i}\left\{x_{i j} T\left(y_{i j}\right)\right\}$ (by (15) again). (Note that by interconnectivity (V) we do not have to worry about $P_{i j}\left(x_{i j} y_{i j}\right)$.) For (iv) with, $y_{i i}=P_{i i}\left(y_{i j}^{2}\right)$ by interconnectivity (I) we have $T\left(x_{i i} y_{i i}\right)=T\left(x_{i i} y_{i j}^{2}\right)=$ $T\left(P_{i i}\left\{\left(x_{i i} \circ y_{i j}\right) y_{i j}\right\}\right)$ (by (20)) $=P_{i i}\left\{T\left(x_{i i} \circ y_{i j}\right) y_{i j}\right\}$ (by (iii)) $=P_{i i}\left\{\left(T\left(x_{i i}\right) \circ y_{i j}\right) y_{i j}\right\}$ (by (ii)) $=T\left(x_{i i}\right) y_{i j}^{2}$ (by (20) again) $=T\left(x_{i i}\right) y_{i i}$ and dually.

Thus $T$ is an element $\gamma$ of the centroid $\Gamma(\mathfrak{U})$. We call $\gamma$ the indicator of $\mathfrak{U}$ (relative to the given interconnected Peirce decomposition).

Though the indicator always exists, to draw conclusions about the structure of an algebra from $\gamma$ we need to make some technical assumptions about $\gamma$ being "well 
behaved" (compare [3, p. 190]). Note that since $L_{e_{i}} R_{e_{i}}=\gamma I$ and $L_{e_{i}}+R_{e_{i}}=I$ we have $L_{e_{i}}^{2}-L_{e_{i}}+\gamma I=0$ on any $\mathfrak{A}_{i j}$. If $\gamma$ has the form $\gamma=\lambda(1-\lambda)$, this implies $\left\{L_{e_{i}}-\lambda I\right\}\left\{L_{e_{i}}-(1-\lambda) I\right\}=0$. If we were working in a vector space over a field this would say $\mathfrak{A}_{i j}$ is the sum of "eigenspaces" $\mathfrak{A}_{i j}=\mathfrak{U}_{i j}^{[\lambda]}+\mathfrak{U}_{i j}^{[1-\lambda]}$ where

$$
\mathfrak{A}_{i j}^{[\lambda]}=\left\{x_{i j} \in \mathfrak{A}_{i j} \mid e_{i} x_{i j}=\lambda x_{i j}\right\}=\mathfrak{A}_{j i}^{[1-\lambda]}
$$

(at least if $\lambda \neq 1-\lambda$; if $\lambda=\frac{1}{2}$, corresponding to $\gamma=\frac{1}{4}$, we would only know $\left(L_{e_{i}}-\frac{1}{2} I\right)^{2}$ $=0$, whereas $\mathfrak{A}_{i j}=\mathfrak{A}_{i j}^{[1 / 2]}$ would mean $L_{e_{i}}-\frac{1}{2} I=0$ on $\left.\mathfrak{A}_{i j}\right)$. We say the indicator is regular if there is a free extension $\Omega$ of $\Phi$ such that in $\widetilde{\mathfrak{U}}=\mathfrak{A} \otimes_{\Gamma} \Omega$ we have

(R) $\tilde{\mathfrak{A}}_{i j}=\tilde{\mathfrak{A}}_{i j}^{[\lambda]}+\tilde{\mathfrak{A}}_{i j}^{[1-\lambda]}$

where $\lambda \in \Omega$ satisfies $\lambda(1-\lambda)=\gamma$ and, if $\gamma \neq \frac{1}{4}, 2 \lambda-1$ is invertible.

At first glance these seem like severe restrictions on $\gamma$. Let us see that they are satisfied in most cases of interest. We first need a

LEMMA 7 [3, pp. 194-195]. If $\mathfrak{B}=\sum \mathfrak{B}_{i j}$ where $\mathfrak{B}_{i j}=\mathfrak{A}_{i j}^{[\lambda]}+\mathfrak{A}_{i j}^{[1-\lambda]}(i \neq j)$ and $\mathfrak{B}_{i i}=P_{i i}\left(\mathfrak{B}_{i j} \circ \mathfrak{U}_{i j}\right)(i \neq j)$ then $\mathfrak{B}$ is an ideal in the $n$-interconnected algebra $\mathfrak{A}(n \geqq 3)$.

Proof. We first show $\mathfrak{B}_{i i}$ is well defined. If $i, j, k \neq$, we have $P_{i i}\left(\mathfrak{B}_{i j} \circ \mathfrak{A}_{i j}\right)$ $=P_{i i}\left(\mathfrak{B}_{i j} \circ\left(\mathfrak{U}_{j k} \circ \mathfrak{A}_{k i}\right)\right)$ (by interconnectivity $\left.\left(\mathrm{I}^{\prime}\right)\right)=P_{i i}\left\{\left(\mathfrak{B}_{i j} \circ \mathfrak{A}_{j k}\right) \circ \mathfrak{A}_{k i}\right\} \subset P_{i i}\left(\mathfrak{B}_{i k} \circ \mathfrak{A}_{k i}\right)$ so $P_{i \imath}\left(\mathfrak{B}_{i j} \circ \mathfrak{A}_{i j}\right)=P_{i i}\left(\mathfrak{B}_{i k} \circ \mathfrak{A}_{i k}\right)$ all have a common value $\mathfrak{B}_{i i}$. Here we have used $\mathfrak{B}_{i j} \circ \mathfrak{A}_{j k} \subset \mathfrak{B}_{i k}$ since $E_{\mathfrak{Q}_{j k}}$ commute with $L_{e_{i}}-\lambda I$ and hence leave $\operatorname{Ker}\left(L_{e_{\mathfrak{t}}}-\lambda I\right)$ invariant.

By (14) we have $\mathfrak{A}_{i k} \mathfrak{B}_{i j}+\mathfrak{B}_{i j} \mathfrak{A}_{i k} \subset \mathfrak{B}_{k j}$ for $k \neq j$, and by (15)' $\mathfrak{A}_{i j} \mathfrak{B}_{i j}+\mathfrak{B}_{i j} \mathfrak{A}_{i j}$ $\subset \mathfrak{B}_{i i}+\mathfrak{B}_{j j}$ (again by interconnectivity (V) we do not have to worry about $\left.P_{i j}\left(\mathfrak{U}_{i j} \mathfrak{B}_{i j}\right)\right)$. Proposition 1 then guarantees $\mathfrak{A}_{i i} \mathfrak{B}_{i i}+\mathfrak{B}_{i i} \mathfrak{A}_{i i} \subset \mathfrak{B}_{i i}$. The only remaining thing to check is that $\mathfrak{A}_{i j} \mathfrak{B}_{i i}+\mathfrak{B}_{i i} \mathfrak{A}_{i j} \subset \mathfrak{B}_{i j}$. By (14)' it will suffice if $\mathfrak{A}_{i j} \circ \mathfrak{B}_{i i} \subset \mathfrak{B}_{i j}$. Since $n \geqq 3$ we have $\mathfrak{A}_{i j} \circ \mathfrak{B}_{i i}=\mathfrak{A}_{i j} \circ P_{i i}\left(\mathfrak{A}_{i k} \circ \mathfrak{B}_{i k}\right)($ for $k \neq i, j)=\mathfrak{A}_{i j} \circ\left(\mathfrak{A}_{i k} \circ \mathfrak{B}_{i k}\right)$ $=\left(\mathfrak{A}_{i j} \circ \mathfrak{A}_{i k}\right) \circ \mathfrak{B}_{i k}+\left(\mathfrak{A}_{i j} \circ \mathfrak{B}_{i k}\right) \circ \mathfrak{A}_{i k}$ (using linearized $\left.(20)^{\prime}\right) \subset \mathfrak{A}_{j k} \circ \mathfrak{B}_{i k}+\mathfrak{B}_{j k} \circ \mathfrak{A}_{i k}$ $\subset \mathfrak{B}_{i j}$.

COROLlaRY. If $\mathfrak{A}$ is simple and n-interconnected for $n \geqq 3$ then for any $\lambda \in \Gamma$ either all $\mathfrak{A U}_{i j}^{[\lambda]}=\mathfrak{Q C}_{i j}^{[1-\lambda]}=0$ or else all $\mathfrak{A}_{i j}^{[\lambda]}+\mathfrak{A}_{i j}^{[1-\lambda]}=\mathfrak{A}_{i j}$.

This shows us first of all that the indicator of a simple algebra $\mathfrak{A}$ is always regular. Indeed, $\mathfrak{A}$ is a vector space over the field $\Gamma(\mathfrak{U})$ in this situation, and if we take any field extension $\Omega$ in which $\lambda(1-\lambda)=\gamma$ has a root, necessarily $2 \lambda-1$ will be invertible if $\lambda \neq \frac{1}{2}\left(\gamma \neq \frac{1}{4}\right)$. We remarked that (R) holds automatically if $\lambda \neq \frac{1}{2}$, while if $\lambda=\frac{1}{2}$ then $\left(L_{e_{i}}-\frac{1}{2} I\right)^{2}=0$ shows $\tilde{\mathfrak{A}}_{i j}^{[1 / 2]} \neq 0$, so by the corollary (applied to the central simple algebra $\tilde{\mathfrak{A}})$ we know $\tilde{\mathfrak{A}}_{i j}=\tilde{\mathfrak{A}}_{i j}^{[1 / 2]}$.

It also shows us that $(R)$ is equivalent to

$(\mathrm{R})^{\prime} e_{i} \in P_{i i}\left\{\tilde{\mathfrak{A}}_{i j} \circ \tilde{\mathfrak{B}}_{i j}\right\}\left(\tilde{\mathfrak{B}}_{i j}=\tilde{\mathfrak{A}}_{i j}^{[\lambda]}+\tilde{\mathfrak{U}}_{i j}^{[1-\lambda]}\right)$

for unital algebras, because this condition guarantees $1=\sum e_{i}$ belongs to the ideal $\tilde{\mathfrak{B}}$ and therefore $\tilde{\mathfrak{B}}=\tilde{\mathfrak{A}}$. If $\mathfrak{A}$ is a unital subalgebra of a unital algebra $\mathfrak{E}$ then $\mathfrak{E}$ will inherit $(R)^{\prime}$ from $\mathfrak{A}$. In particular, if $\mathfrak{M}$ is a unital bimodule for $\mathfrak{A}$ then $(R)^{\prime}$ on 
$\mathfrak{U}$ implies $(\mathbf{R})^{\prime}$ on the split null extension $\mathfrak{E}=\mathfrak{A} \oplus \mathfrak{M}$. In general there need be no relation between the centroids $\Gamma(\mathfrak{H})$ and $\Gamma(\mathfrak{E})$, since $\mathfrak{M}$ need only be a $\Phi$-module, but if we assume $\mathfrak{A}$ is central, $\Gamma(\mathfrak{U})=\Phi$, then $\&$ is necessarily a $\Gamma$-module; if $\Omega$ is the free extension of $\Gamma$ required by regularity of $\mathfrak{A}$ then $\Sigma=\Gamma(\mathfrak{E}) \otimes_{\Gamma} \Omega$ is a suitable free extension of $\Gamma(\mathbb{E})$. Thus if $\mathfrak{A}$ is central, $\mathbb{E}$ inherits not only (R) but regularity from $\mathfrak{A}$.

Now we investigate what sort of information the indicator gives us about an algebra.

LEMMA 8 [3, p. 191]. If $\mathfrak{A}$ is $n$-interconnected for $n \geqq 3$ with regular indicator $\gamma=\frac{1}{4}$ then $\mathfrak{A}$ is commutative.

Proof. We must prove $[x, y]=0$ for $x, y$ in the Peirce spaces. If $x \in \mathfrak{A}_{i j}$ we can find $k \neq i, j$ by our hypothesis $n \geqq 3$, so, relative to $e=e_{k}, x$ belongs to $\mathfrak{A}_{0}$.

If $y \in \mathfrak{A}_{1}$ then $\left[x_{0}, y_{1}\right]=0$ by Peirce orthogonality.

If $y \in \mathfrak{A}_{1 / 2}$ then $\left[x_{0}, y_{1 / 2}\right]=x_{0} y_{1 / 2}-y_{1 / 2} x_{0}=\left(x_{0} \circ y_{1 / 2}\right) e-e\left(y_{1 / 2} \circ x_{0}\right)=\left[x_{0} \circ y_{1 / 2}, e\right]$ by (14), so it is enough if $\left[e, \mathfrak{A}_{1 / 2}\right]=0$. If we are assuming $\gamma=\frac{1}{4}$ is regular we have $L_{e}=R_{e}=\frac{1}{2} I$ on $\mathfrak{A}_{1 / 2}$, so $\left[e, \mathfrak{A}_{1 / 2}\right]=0$.

Since $\mathfrak{A}_{0}$ is spanned by $\boldsymbol{P}_{0}\left(\mathfrak{H}_{1 / 2}^{2}\right)$ by interconnectivity, it suffices to consider $y_{0}=P_{0}\left(y_{1 / 2}^{2}\right)$ in proving $\left[x_{0}, y_{0}\right]=0$. But then $\left[x_{0}, y_{0}\right]=\left[x_{0}, y_{1 / 2}^{2}\right]=\left[x_{0}, y_{1 / 2}\right] \circ y_{1 / 2}$ (using $\left.(1)^{\prime}\right)$ and this vanishes by the previous case.

Thus $[x, y]=0$ in all cases.

When $\gamma=0$ we have no need of regularity.

LEMMA 9 [3, p. 191]. If $\mathfrak{A}$ is $n$-interconnected for $n \geqq 3$ with indicator $\gamma=0$ then $\mathfrak{A}$ is associative.

Proof. We must prove $[x, \mathfrak{A}, y]=0$ for all $x, y$ in the various Peirce spaces. If $x \in \mathfrak{A}_{i j}$ we can find $k \neq i, j$ so (relative to $e=e_{k}$ ) we may again assume $x \in \mathfrak{A}_{0}$. (As usual, if $k=0$ we interpret this suitably-that $x \in \mathfrak{A}_{1}$ relative to $e=e_{1}+\cdots+e_{n-1}$.) We consider the three cases $y \in \mathfrak{A}_{1}, y \in \mathfrak{A}_{1 / 2}, y \in \mathfrak{A}_{0}$. Note that $e \mathfrak{A}_{1 / 2} e=\gamma \mathfrak{A}_{1 / 2}=0$ by definition of $\gamma$.

If $y \in \mathfrak{A}_{1}$ then $\left[x_{0}, \mathfrak{A}, y_{1}\right]=\left[R_{y_{1}}, L_{x_{0}}\right] \mathfrak{A}=0$ by (16).

If $y \in \mathfrak{A}_{1 / 2}$ then $\left[x_{0}, \mathfrak{A}, y_{1 / 2}\right]=\left[x_{0}, \mathfrak{A}, e \circ y_{1 / 2}\right]=-\left[e, \mathfrak{A}, x_{0} \circ y_{1 / 2}\right]-\left[y_{1 / 2}, \mathfrak{A}, x_{0} \circ e\right]$ (by linearized $\left.(2)^{\prime}\right)=-\left[e, \mathfrak{A}, x_{0} \circ y_{1 / 2}\right] \subset\left[e, \mathfrak{A}, \mathfrak{A}_{1 / 2}\right]$ so we need only prove $\left[e, \mathfrak{A}, y_{1 / 2}\right]=0$. Now $\left[e, \mathfrak{A}_{0}, y_{1 / 2}\right]=-e\left(\mathfrak{A}_{0} y_{1 / 2}\right)=-e\left(\mathfrak{A}_{0} \circ y_{1 / 2}\right) e($ by $(14))=0$ and similarly $\left[e, \mathfrak{A}_{1}, y_{1 / 2}\right]=\mathfrak{A}_{1} y_{1 / 2}-e\left(\mathfrak{A}_{1} y_{1 / 2}\right)=\left(\mathfrak{A}_{1} y_{1 / 2}\right) e=e\left(\mathfrak{A}_{1} \circ y_{1 / 2}\right) e=0$. Also,

$$
\begin{aligned}
{\left[e, \mathfrak{A}_{1 / 2}, y_{1 / 2}\right] } & =\left(e \mathfrak{A}_{1 / 2}\right) y_{1 / 2}-e\left(\mathfrak{A}_{1 / 2} y_{1 / 2}\right) \\
& =P_{1}\left\{e\left(e \mathfrak{A}_{1 / 2}\right) \circ y_{1 / 2}\right\}+P_{0}\left\{\left(e \mathfrak{A}_{1 / 2}\right) e \circ y_{1 / 2}\right\}-P_{1}\left\{e \mathfrak{A}_{1 / 2} \circ y_{1 / 2}\right\} \text { (by (15); }
\end{aligned}
$$

note again by $(\mathrm{V})$ we can forget about $\left.P_{1 / 2}\left(\mathfrak{A}_{1 / 2}^{2}\right)\right)=P_{0}\left\{e \mathfrak{A}_{1 / 2} e \circ y_{1 / 2}\right\}-P_{1}\left\{e \mathfrak{A}_{1 / 2} e \circ y_{1 / 2}\right\}$ $=0$. Thus $\left[e, \mathfrak{A}, y_{1 / 2}\right]=0$. 
If $y \in \mathfrak{A}_{0}$ then $\left[x_{0}, \mathfrak{A}_{1}, y_{0}\right]=0$ by Peirce orthogonality, and

$$
\begin{aligned}
{\left[x_{0}, \mathfrak{A}_{1 / 2}, y_{0}\right] } & =\left(x_{0} \mathfrak{A}_{1 / 2}\right) y_{0}-x_{0}\left(\mathfrak{A}_{1 / 2} y_{0}\right)=\left(x_{0} \circ \mathfrak{A}_{1 / 2} e\right) y_{0}-x_{0}\left(e \mathfrak{A}_{1 / 2} \circ y_{0}\right) \\
& =e\left(x_{0} \circ \mathfrak{A}_{1 / 2} e\right) \circ y_{0}-x_{0} \circ\left(e \mathfrak{A}_{1 / 2} \circ y_{0}\right) e \\
& =\left(x_{0} \circ e \mathfrak{A}_{1 / 2} e\right) \circ y_{0}-x_{0} \circ\left(e \mathfrak{A}_{1 / 2} e \circ y_{0}\right)=0 \quad \text { by (14). }
\end{aligned}
$$

For $\left[x_{0}, \mathfrak{A}_{0}, y_{0}\right]$ we use the interconnectivity; as $\mathfrak{A}_{0}=P_{0}\left(\mathfrak{U}_{1 / 2}^{2}\right)$ it is enough to take $y_{0}=P_{0}\left(y_{1 / 2}^{2}\right)$. But $\left[x_{0}, \mathfrak{A}_{0}, P_{0}\left(y_{1 / 2}^{2}\right)\right]=\left[x_{0}, \mathfrak{A}_{0}, y_{1 / 2}^{2}\right]($ by $(16))=-\left[y_{1 / 2}, \mathfrak{A}_{0}, x_{0} \circ y_{1 / 2}\right]$ (by $\left.(2)^{\prime}\right)$, so we need only show $\left[y_{1 / 2}, \mathfrak{A}_{0}, z_{1 / 2}\right]=0$. Here

$$
\begin{aligned}
{\left[y_{1 / 2}, \mathfrak{A}_{0}, z_{1 / 2}\right]=} & \left(y_{1 / 2} \mathfrak{A}_{0}\right) z_{1 / 2}-y_{1 / 2}\left(\mathfrak{A}_{0} z_{1 / 2}\right) \\
= & P_{1}\left\{\left(y_{1 / 2} \mathfrak{A}_{0}\right) \circ z_{1 / 2} e-e y_{1 / 2} \circ\left(\mathfrak{A}_{0} z_{1 / 2}\right)\right\} \\
& +P_{0}\left\{\left(y_{1 / 2} \mathfrak{A}_{0}\right) e \circ z_{1 / 2}-y_{1 / 2} \circ e\left(\mathfrak{A}_{0} z_{1 / 2}\right)\right\} \\
= & P_{1}\left\{\left(e y_{1 / 2} \circ \mathfrak{A}_{0}\right) \circ z_{1 / 2} e-e y_{1 / 2} \circ\left(\mathfrak{U}_{0} \circ z_{1 / 2} e\right)\right\} \\
& +P_{0}\left\{e\left(y_{1 / 2} \circ \mathfrak{A}_{0}\right) e \circ z_{1 / 2}-y_{1 / 2} \circ e\left(\mathfrak{A}_{0} \circ z_{1 / 2}\right) e\right\} \\
= & 0 \quad \text { by }(14),(15),
\end{aligned}
$$

and the relation $P_{1}\left\{\left(a_{1 / 2} \circ b_{0}\right) \circ c_{1 / 2}\right\}=\left\{a_{1 / 2} b_{0} c_{1 / 2}\right\}=P_{1}\left\{a_{1 / 2} \circ\left(b_{0} \circ c_{1 / 2}\right)\right\}$ which holds in any quadratic Jordan algebra. Thus $\left[x_{0}, \mathfrak{A}, y_{0}\right]=0$. This completes the proof of associativity.

For any $\mu \in \Phi$ we can define a new algebra structure on $\mathfrak{A}$, the $\mu$-mutation $\mathfrak{U}^{(\mu)}$, by

$$
x \cdot{ }_{\mu} y=\mu x y+(1-\mu) y x .
$$

The mutation of a noncommutative Jordan algebra is again such, and mutation is transitive in the sense that

$$
\left\{\mathfrak{Q}^{(\mu)}\right\}^{(\lambda)}=\mathfrak{A}^{(\mu \odot \lambda)} \quad(\mu \odot \lambda=2 \mu \lambda-\mu-\lambda+1)
$$

[3, p. 192]. An algebra of the form $\mathfrak{A}=\mathfrak{D}^{(\lambda)}$ for $\mathfrak{D}$ associative is called a split quasiassociative algebra, and an algebra is quasiassociative if it is a form of a split quasiassociative algebra: $\mathfrak{A}_{\Omega}=\mathfrak{D}^{(\lambda)}(\lambda \in \Omega)$ for some free extension $\Omega$ of $\Phi$. The following lemma describes how mutation affects the indicator.

LEMMA 10. If $\mathfrak{A}$ is an n-interconnected algebra with indicator $\gamma$ then for any $\mu \in \Phi$ the mutation $\mathfrak{Q}^{(\mu)}$ is also n-interconnected with indicator $\gamma^{(\mu)}=(4 \gamma-1) \mu^{2}$ $-(4 \gamma-1) \mu+\gamma=(2 \mu-1)^{2} \gamma+\mu(1-\mu)$.

Proof. $\mathfrak{A}^{(\mu)}$ has the same interconnected Peirce decomposition as $\mathfrak{A}$ does relative to the idempotents $e_{i}$ since $\mathfrak{U}^{(\mu)+}=\mathfrak{A}^{+}$. On $\mathfrak{A}_{i j}$ for $i \neq j$ we have $L_{e_{i}}^{(\mu)}=\mu L_{e_{i}}+(1-\mu) R_{e_{i}}$ $=\mu L_{e}+(1-\mu)\left(I-L_{e_{\mathrm{i}}}\right)=(2 \mu-1) L_{e_{i}}+(1-\mu) I$ so

$$
\begin{aligned}
\gamma^{(\mu)} & =L_{e_{i}}^{(\mu)}\left\{I-L_{e_{i}}^{(\mu)}\right\}=\left\{(1-\mu) I+(2 \mu-1) L_{e_{i}}\right\}\left\{\mu I-(2 \mu-1) L_{e_{i}}\right\} \\
& =\mu(1-\mu) I+(2 \mu-1)^{2} L_{e_{i}}-(2 \mu-1)^{2} L_{e_{i}}^{2} \\
& =\mu(1-\mu) I+(2 \mu-1)^{2} \gamma=(4 \gamma-1) \mu^{2}-(4 \gamma-1) \mu+\gamma .
\end{aligned}
$$


From these results we obtain

THEOREM 4. If $\mathfrak{A}$ is an $n$-interconnected algebra, $n \geqq 3$, with regular indicator, then $\mathfrak{A}$ is either a commutative Jordan algebra of characteristic $\neq 2$ or else a quasiassociative algebra.

Proof. If the indicator is $\gamma=\frac{1}{4}$ then clearly $\Gamma$ has characteristic $\neq 2$, and if $\gamma$ is regular by Lemma $8 \mathfrak{A}$ must be commutative.

If $\gamma \neq \frac{1}{4}$ then by regularity there is a free extension $\Omega$ of $\Gamma$ and $\lambda \in \Omega$, $\mu=\lambda /(2 \lambda-1) \in \Omega$ with $\lambda(1-\lambda)=\gamma$, so by Lemma 10 the mutation $\mathfrak{A}_{\Omega}^{(\mu)}$ has indicator $\gamma^{(\mu)}=(2 \mu-1)^{2} \gamma+\mu(1-\mu)=\gamma(2 \lambda-1)^{-2}+\lambda(\lambda-1)(2 \lambda-1)^{-2}=0$. But then $\mathfrak{A}_{\Omega}^{(\mu)}=\mathfrak{D}$ is associative by Lemma 9 , and because $\mu \odot \lambda=1$ we have $\mathfrak{A}_{\Omega}=\mathfrak{A}_{\Omega}^{(1)}=\mathfrak{A}_{\Omega}^{(\mu \odot \lambda)}$ $=\left\{\mathfrak{U}_{\Omega}^{(\mu)}\right\}^{(\lambda)}=\mathfrak{D}^{(\lambda)}$ by (23). This means $\mathfrak{A}_{\Omega}$ is split quasiassociative and $\mathfrak{A}$ plain quasiassociative over its centroid.

We noted that the indicator was regular in the case of simple algebras. Thus Theorems 3 and 4 lead to one of the main results of this paper:

THEOREM 5. A simple noncommutative Jordan algebra with two nonzero orthogonal idempotents whose sum is not 1 is either a commutative Jordan algebra over a field of characteristic $\neq 2$ or a quasiassociative algebra.

We stress once more that in this result we do not assume the existence of a unit element, we impose no finiteness restrictions, and we allow an arbitrary ring of scalars - the theorem is completely general. Moreover, the result extends to unital bimodules since we remarked that regularity extends from unital central algebras to the split null extension.

THEOREM 6. Let $\mathfrak{M}$ be a unital bimodule for a central $n$-interconnected unital noncommutative Jordan algebra $\mathfrak{A}(n \geqq 3)$ with regular indicator. Then either $\mathfrak{M}$ is a commutative Jordan bimodule for the commutative Jordan algebra $\mathfrak{A}$ or $\mathfrak{M}$ is a quasiassociative bimodule for the quasiassociative algebra $\mathfrak{A}$.

Here $\mathfrak{M}$ is a split quasiassociative bimodule for $\mathfrak{A}=\mathfrak{D}^{(\lambda)}$ if $\mathfrak{M}=\mathfrak{R}^{(\lambda)}$ for $\mathfrak{R}$ an associative bimodule for $\mathfrak{D}$, and is a quasiassociative bimodule if it becomes split under some free extension. In particular,

THEOREM 7. Let $\mathfrak{M}$ be a unital bimodule for a central simple unital noncommutative Jordan algebra $\mathfrak{A}$ which has three nonzero supplementary orthogonal idempotents. Then either $\mathfrak{M}$ is a commutative Jordan bimodule for the commutative Jordan algebra $\mathfrak{A}$ or $\mathfrak{M}$ is a quasiassociative bimodule for the quasiassociative algebra $\mathfrak{A}$.

5. Connected idempotents. We consider now a more restrictive kind of connectivity between idempotents, one which does not hold in all simple algebras but does hold for those with certain finiteness conditions.

Two orthogonal idempotents $e_{1}, e_{2}$ are connected if there is an element $u_{12}$ in the Peirce space $\mathfrak{A}_{12}$ which is invertible in the Peirce subalgebra $\mathfrak{A}_{11}+\mathfrak{A}_{12}+\mathfrak{A}_{22}$. Since $u_{12}$ is invertible if and only if $u_{12}^{2}=u_{11}+u_{22}$ is $\left(u_{i i} \in \mathfrak{A}_{i i}\right)$, we see $u_{12}$ is invertible if 
and only if the $u_{i i}$ are invertible in $\mathfrak{A}_{i i}$. We say $e_{1}, e_{2}$ are strongly connected if there is $u_{12} \in \mathfrak{A}_{12}$ with $u_{12}^{2}=e_{1}+e_{2}$. These notions coincide with those for the quadratic Jordan algebra $\mathfrak{A}^{+}$. The difference between connectivity and interconnectivity is that whereas in connectivity we have $e_{i}=P_{i i}\left(x_{i j} \circ y_{i j}\right)$ (using (20) with $x_{1 / 2}=u_{12}$, $\left.y_{i}=u_{i i}^{-1}\right)$, in interconnectivity $e_{i} \in P_{i i}\left(\mathfrak{U}_{i j} \circ \mathfrak{A}_{i j}\right)$ is only a sum of such elements $P_{i i}\left(x_{i j} \circ y_{i j}\right)$. A family $\left\{e_{i}\right\}$ of orthogonal idempotents is connected or strongly connected if each pair $e_{i}, e_{j}$ is. We say $\mathfrak{A}$ is $n$-connected (or strongly n-connected) if there exists a connected (or strongly connected) family $e_{1}, \ldots, e_{n}$ of supplementary orthogonal idempotents.

From the Jordan theory [2, p. 1077] we know that for any $n$-connected quadratic Jordan algebra $\Im, n \geqq 3$, we have an epimorphism of $\Im$ onto a Jordan algebra $\mathfrak{S}\left(\mathfrak{D}_{n}, \mathfrak{D}_{0}, \gamma\right)$ of matrices in $\mathfrak{D}_{n}$ symmetric with respect to the involution $X \rightarrow X^{*}$ $=\gamma^{-1} \bar{X}^{t} \gamma$ and having diagonal entries in $\mathfrak{D}_{0}$; here $\mathfrak{D}$ is alternative with involution (associative if $n \geqq 4$ ), $\mathfrak{D}_{0}$ an ample subspace (i.e. subspace of symmetric elements in the nucleus containing 1 and such that $\delta \mathscr{D}_{0} \delta \subset \mathfrak{D}_{0}$ for all $\left.\delta \in \mathfrak{D}\right)$, and $\gamma=\operatorname{diag}\left\{\gamma_{1}, \ldots, \gamma_{n}\right\}$ is a diagonal matrix where the $\gamma_{i} \in \mathscr{D}_{0}$ are invertible. (For a strongly connected algebra we can take $\gamma=1$.) The kernel of this homomorphism is the extreme radical $\mathfrak{R}=\sum \mathfrak{\Re}_{i i}$, where $\mathfrak{\Re}_{i i} \circ \mathfrak{\Im}_{i j}=0$.

If we define matrices in $\mathfrak{S}_{\mathcal{E}}\left(\mathfrak{D}_{n}, \mathfrak{D}_{0}, \gamma\right)$ by

$$
\begin{aligned}
& \alpha[i i]=\alpha \gamma_{i} e_{i i} \quad\left(\alpha \in \mathscr{D}_{0}\right) \\
& a[i j]=a \gamma_{j} e_{i j}+\bar{a} \gamma_{i} e_{j i}=\bar{a}[j i] \quad(a \in \mathfrak{D}, i \neq j)
\end{aligned}
$$

in terms of the matrix units $e_{i j}$ in $\mathfrak{D}_{n}$ then the Peirce decomposition relative to the diagonal idempotents $e_{i}=\gamma_{i}^{-1}[i i]=e_{i i}$ is

$$
\mathfrak{A}_{i i}=\mathfrak{D}_{0}[i i], \quad \mathfrak{A}_{i j}=\mathfrak{D}[i j]=\mathfrak{D}[j i] \quad(i \neq j) .
$$

Multiplication obeys the following rules:

(a) $\alpha[i i] \circ b[i j]=\alpha \gamma_{i} b[i j], b[i j] \circ \alpha[j j]=b \gamma_{j} \alpha[i j]$,

(b) $a[i j] \circ b[j k]=a \gamma_{j} b[i k]$

(c) $a[i j]^{2}=a \gamma_{j} \bar{a}[i i]+\bar{a} \gamma_{i} a[j j], a[i j] \circ b[i j]=t\left(a \gamma_{j} \bar{b}\right)[i i]+t\left(\bar{a} \gamma_{i} b\right)[j j]$ for $t(a)=a+\bar{a}$.

With connectivity replacing interconnectivity, we can improve on Theorem 4 as follows:

COORDINATIZATION THEOREM. If $\mathfrak{A}$ is an n-connected noncommutative Jordan algebra, $n \geqq 3$, then $\mathfrak{A}$ is isomorphic to either

(I) a commutative Jordan algebra $\mathfrak{S}\left(\mathfrak{D}_{n}, \gamma\right)$ of characteristic $\neq 2$,

(II) an algebra $\mathfrak{S}_{\mathrm{g}}\left(\mathfrak{D}_{n}, \gamma\right)^{(\lambda)}$ where $\lambda \neq \frac{1}{2}$ with $\lambda+\bar{\lambda}=1$ belongs to the center of $\mathfrak{D}$ and $(2 \lambda-1)[\mathfrak{D}, \mathfrak{D}, \mathfrak{D}]=0$,

where $\mathfrak{D}$ is associative if $n \geqq 4$ and for $n \geqq 3$ is alternative with involution whose symmetric elements lie in the nucleus.

Proof. [8, p. 3]. The $e_{i}$ are still connected in $\mathfrak{A}^{+}$, so we know we have an epimorphism of $\mathfrak{S}=\mathfrak{U}^{+}$onto $\mathfrak{S}_{\mathfrak{S}}\left(\mathfrak{D}_{n}, \mathfrak{D}_{0}, \gamma\right)$ as above. Since $\mathfrak{U}$ is connected it is inter- 
connected and unital, so by interconnectivity (III') the relation $\mathfrak{\Re}_{i i} \circ \mathfrak{A}_{i j}=0$ for the extreme radical $\mathfrak{R}$ shows $\mathfrak{R}_{i \mathfrak{i}}=0, \mathfrak{R}=0$, and the epimorphism is actually an isomorphism. We identify $\mathfrak{U}^{+}$with $\mathfrak{S}\left(\mathfrak{D}_{n}, \mathfrak{D}_{0}, \gamma\right)$.

Since $e_{i} \mathfrak{A}_{i j} \subset \mathfrak{A}_{i j}$ we can define linear transformations $L_{i j}$ on $\mathfrak{D}$ by

$$
L_{i j}(a)[i j]=L_{e_{i}}\{a[i j]\}=R_{e_{j}}\{a[i j]\} .
$$

Since $L_{e_{i}}$ commutes with $V_{y_{j k}}$, for $y_{j k}=\gamma_{j}^{-1} b[j k]$ we see by (b) that

$$
L_{i j}(a) b=L_{i k}(a b)
$$

and similarly since $e_{k}$ commutes with $L_{x_{i j}}$ for $x_{i j}=a \gamma_{j}^{-1}[i j]$ that

$$
L_{i k}(a b)=a L_{j k}(b) \text {. }
$$

Setting $b=e$ shows $L_{i j}=L_{i k}$, and $a=e$ shows $L_{i k}=L_{j k}$, so all $L_{i j}$ have a common value $L$. Since $L(a b)=L(a) b=a L(b), L(a)=\lambda a=a \lambda$ for $\lambda=L(e)$ in the center of $\mathfrak{D}$ :

$$
e_{i}\{a[i j]\}=\lambda a[i j] .
$$

Since $e[i j]=e_{i}\{e[i j]\}+e_{j}\{e[i j]\}=e_{i}\{e[i j]\}+e_{j}\{\bar{e}[j i]\}=\lambda e[i j]+\lambda \bar{e}[j i]=\{\lambda e+e \bar{\lambda}\}[i j]=$ $(\lambda+\bar{\lambda})[i j]$ we see $\lambda+\bar{\lambda}=e$.

Already this allows us to dispense with $\mathscr{D}_{0}$ : we claim $\mathscr{D}_{0}$ is just the set $\mathfrak{S}(\mathfrak{D})$ of all symmetric elements of $\mathfrak{D}$, since any $\delta=\delta$ has $\delta=(\lambda+\bar{\lambda}) \delta=\lambda \delta+\delta \bar{\lambda}=\lambda \delta+\bar{\delta}$ $=t(\lambda \delta)$, a trace, and $\mathfrak{D}_{0}$ contains all traces.

By Peirce orthogonality, multiplication in $\mathfrak{A}$ will be completely determined by the following multiplication table:

(i) $\alpha[i i] b[i j]=\lambda \alpha \gamma_{i} b[i j], b[i j] \alpha[i i]=\lambda \alpha \gamma_{i} b[i j]$,

(ii) $a[i j] b[j k]=\lambda a \gamma_{j} b[i k]$,

(iii) $a[i j] b[i j]=t\left(\lambda a \gamma_{j} \bar{b}\right)[i i]+t\left(\lambda \bar{a} \gamma_{i} b\right)[j j]$,

(iv) $\alpha[i i] \beta[i i]=t\left(\lambda \alpha \gamma_{i} \beta\right)[i i]$,

for $i, j, k \neq$ and $\alpha, \beta \in \mathfrak{D}_{0}, a, b, \in \mathfrak{D}$.

For (i) we apply (a), (14), and (*); similarly (ii) follows from (b), (14), and (*). By interconnectivity (V) there is no $i j$-component in $a[i j] b[i j]$, so (iii) follows from (c), (15), and (*). Finally, for (iv) we use connectivity in the form

$$
\begin{aligned}
\alpha[i i] \beta[i i] & =\alpha[i i]\left\{U_{\gamma_{j}^{-1}[i j]} \beta[j j]\right\} \\
& =-\beta[j j]\left\{U_{\gamma_{j}^{-1}[i j]} \alpha[i i]\right\}+\left\{U_{\alpha[i i], \beta[j j]} \gamma_{j}^{-1}[i j]\right\} \gamma_{j}^{-1}[i j] \quad \text { (linearizing (6)), }
\end{aligned}
$$

and the $\mathfrak{A}_{i i}$ component of this expression is

$$
\begin{aligned}
P_{i i}\left\{\left\{\alpha[i i] \circ\left(\beta[j j] \circ \gamma_{j}^{-1}[i j]\right)\right\} \gamma_{j}^{-1}[i j]\right\} & =P_{i\{}\left\{\alpha \gamma_{i} \beta[i j] \gamma_{j}^{-1}[i j]\right\} \\
& =t\left(\lambda \alpha \gamma_{i} \beta\right)[i i] \text { by (iii). }
\end{aligned}
$$

If $\gamma=\frac{1}{4}$ then $\mathfrak{A}=\mathfrak{S}\left(\mathfrak{D}_{n}, \gamma\right)$ is just the usual commutative Jordan algebra as in (I). If $\gamma \neq \frac{1}{4}$ then the formulas (i)-(iv) are those of multiplication in the mutation $\mathfrak{S}\left(\mathfrak{D}_{n}, \gamma\right)^{(\lambda)}$, so $\mathfrak{A}=\mathfrak{S}_{(}\left(\mathfrak{D}_{n}, \gamma\right)^{(\lambda)}$. For $a, b, c \in \mathfrak{D}$ we have $\lambda[a, b, c]=[\lambda a, b, c]$ $=-[\bar{\lambda} \bar{a}, b, c]=-\lambda[\bar{a}, b, c]=\bar{\lambda}[a, b, c]$ because all $a+\bar{a} \in \mathfrak{D}_{0}$ belong to the nucleus. 
Thus $(\lambda-\bar{\lambda})[a, b, c]=0$, and since $\lambda-\bar{\lambda}=\lambda-(1-\lambda)=2 \lambda-1$ we see $(2 \lambda-1)[\mathfrak{D}, \mathfrak{D}, \mathfrak{D}]$ $=0$.

This theorem has an analogue for $n=2$. It is no longer true that an arbitrary 2-connected algebra $\mathfrak{A}$ will have $\mathfrak{A}^{+}=\mathfrak{S}\left(\mathfrak{D}_{2}, \mathfrak{D}_{0}, \gamma\right)$, but for those that do we have

THEOREM 8. If $\mathfrak{U}$ is a noncommutative Jordan algebra such that $\mathfrak{U}^{+}=\mathfrak{S}_{(}\left(\mathfrak{D}_{2}, \mathfrak{D}_{0}, \gamma\right)$ for $\mathfrak{D}$ a unital associative algebra with involution, $\mathfrak{D}_{0}$ an ample subspace which generates $\mathfrak{D}$, then necessarily $\mathfrak{D}_{0}=\mathfrak{S}(\mathfrak{D})$ and $\mathfrak{U}=\mathfrak{H}_{(}\left(\mathfrak{D}_{2}, \gamma\right)^{(\lambda)}$ for some $\lambda$ in the center of $D$ with $\lambda+\lambda=1$.

Proof. We assume $\mathfrak{A}^{+}$has the form $\mathfrak{S}_{(}\left(\mathfrak{D}_{2}, \mathfrak{D}_{0}, \gamma\right)$. Then the Peirce decomposition of $\mathfrak{A}$ and $\mathfrak{A}^{+}$relative to the $e_{i}$ is $\mathfrak{A}_{11}=\mathfrak{D}_{0}[11], \mathfrak{A}_{12}=\mathfrak{D}[12], \mathfrak{A}_{22}=\mathfrak{D}_{0}[22]$ as before. Because of Peirce orthogonality we need only establish the formulas (i), (iii), (iv) as in the previous theorem.

As before we define a linear transformation $L$ on $\mathfrak{D}$ by

$$
L(a)[12]=L_{e_{1}}\{a[12]\} .
$$

Since $L_{e_{1}}$ commutes with $V_{\mathfrak{A}_{i i}}$ by (13), formula (a) shows

$$
L(\alpha b)=\alpha L(b), \quad L(b \alpha)=L(b) \alpha .
$$

Thus $L$ commutes with right and left multiplications by $\alpha \in \mathfrak{D}_{0}$. Since we have no spaces $\mathfrak{A}_{23}$ around to enable us to show $L$ commutes with all left and right multiplications, we have to introduce our assumption that $\mathfrak{D}_{0}$ generates $\mathfrak{D}$ in order to conclude $L$ belongs to the centroid, $L(a)=\lambda a=a \lambda$ for $\lambda=L(e)$ in the center of $\mathfrak{D}$.

The spaces $\mathfrak{A}_{23}$, etc. also gave us easily that $\lambda+\bar{\lambda}=1$; to obtain this in our present situation we observe $\gamma_{2}[11]=P_{11}\left(e[12]^{2}\right)=P_{11}\left\{e_{1}(e[12]) \circ e[12]\right\}=t\left(\lambda \gamma_{2}\right)[11]$ by (c) and (15), thus $\gamma_{2}=\lambda \gamma_{2}+\bar{\gamma}_{2}=(\lambda+\bar{\lambda}) \gamma_{2}$ and $\lambda+\lambda=1$ as desired. From this we get $L_{e_{2}}\{a[21]\}=\lambda a[21]$ and thus attain symmetry. Formulas (i), (iii), (iv) follow exactly as in the previous theorem except for one point: we do not know offhand that $\mathfrak{A}_{12}^{2} \subset \mathfrak{A}_{11}+\mathfrak{A}_{22}$. Here again we make use of the fact that $\mathfrak{D}_{0}$ generates $\mathfrak{D}: \mathfrak{D}$ is spanned by the elements of the form $a=\alpha_{1} \gamma_{1} \alpha_{2} \gamma_{1} \cdots \alpha_{n} \gamma_{1}, b=\beta_{1} \gamma_{1} \cdots \beta_{m} \gamma_{1}$ for $\alpha_{i}, \beta_{i} \in \mathscr{D}_{0}$. Therefore $a[12]=V_{\alpha_{1}[11]} \cdots V_{\alpha_{n}[11]} e[12]$ and $b[12]=V_{\beta_{1}[11]} \cdots V_{\beta_{m}[11]} e[12]$ by (a), so that by (17)

$$
P_{12}\{a[12] b[12]\}=V_{\alpha_{1}[11]} \cdots V_{\alpha_{n}[11]} V_{\beta_{1}[11]} \cdots V_{\beta_{m}[11]} P_{12}\{e[12] e[12]\} .
$$

But $P_{12}\{e[12] e[12]\}=0$ by (GPR 5), so all $P_{12}\{a[12] b[12]\}=0$ as desired.

Note also that $\lambda+\lambda=1$ again implies $\mathfrak{D}_{0}=\mathfrak{S}(\mathfrak{D})$.

This result is due to K. C. Smith [12] in characteristic $\neq 2$, as is the next theorem. Recall that the radical of a quadratic form $Q$ on $\mathfrak{B}$ is the set

$$
\operatorname{Rad} Q=\{v \mid Q(v)=Q(v, \mathfrak{B})=0\},
$$

while the bilinear radical is $\mathfrak{B}^{\perp}=\{v \mid Q(v, \mathfrak{B})=0\}$. The defect of $Q$ is the dimension of $\mathfrak{B}^{\perp} / \operatorname{Rad} Q$. The defect is always zero in characteristic $\neq 2$ since $Q(v)=\frac{1}{2} Q(v, v)$. 
THEOREM 9. If $\mathfrak{A}$ is a noncommutative Jordan algebra such that $\mathfrak{A}^{+}$has the form $\operatorname{Sp}(Q)=\Omega_{1} e_{1}+\Omega_{2} e_{2}+\mathfrak{B}$ of a split quadratic Jordan algebra determined by $a$ quadratic form $Q$ on a faithful $\Omega$-module $\mathfrak{B}$, where the $\Omega_{i}$ are subspaces of $\Omega$ containing 1 such that each $\Omega_{i}$ generates $\Omega$ and that $Q(\mathfrak{B}) \Omega_{i} \subset \Omega_{j}$, then necessarily $\Omega_{1}=\Omega_{2}=\Omega$ and multiplication in $\mathfrak{A}$ is given by

$$
\begin{gathered}
\left(\omega e_{i}\right)\left(\mu e_{i}\right)=\omega \mu e_{i}, \quad\left(\omega e_{i}\right) v=S_{i}(\omega v), \\
v w=Q\left(S_{1} v, w\right) e_{1}+v \times w+Q\left(S_{2} v, w\right) e_{2},
\end{gathered}
$$

where $S_{i}: \mathfrak{B} \rightarrow \mathfrak{B}$ are $\Omega$-linear transformations on $\mathfrak{B}$ and $v \times w$ a bilinear product on $\mathfrak{B}$ satisfying

$$
\begin{aligned}
Q\left(S_{i} v, v\right) & =Q(v), & S_{1}+S_{2} & =I, \\
Q(v \times w, v) & =0, & v \times v & =0 .
\end{aligned}
$$

Necessarily $Q$ has defect zero.

Proof. $\mathfrak{A}$ and $\mathfrak{U}^{+}$have the same Peirce decomposition $\mathfrak{A}_{\mathfrak{i}}=\Omega_{i} e_{i}, \mathfrak{A}_{12}=\mathfrak{B}$ relative to $e_{1}, e_{2}$. Define $S_{i}$ to be the restriction of $L_{e_{i}}$ to $\mathfrak{B}$ (as in the past two theorems), $S_{i} v=e_{i} v$. Clearly $S_{1}+S_{2}=L_{e_{1}}+L_{e_{2}}=L_{1}=I$. By (14), we have $\left(\omega e_{i}\right) v=e_{i}\left\{\left(\omega_{i} e_{i}\right) \circ v\right\}$ $=e_{i}\left\{\omega_{i} v\right\},\left(\omega_{i} e_{i}\right) v=S_{i}\left(\omega_{i} v\right)$. We know $\left(\omega e_{i}\right)\left(\mu e_{i}\right)=\nu e_{i}$ for some $\nu$ in $\Omega$. Then

$$
\begin{aligned}
\nu v & =\left(\nu e_{i}\right) \circ v=\left\{\left(\omega e_{i}\right)\left(\mu e_{i}\right)\right\} \circ v=\left\{V_{v} L_{\omega e_{i}}\right\}\left(\mu e_{i}\right)=\left\{-V_{\omega e_{i}} L_{v}+L_{\omega e_{i}} V_{v}+L_{v} V_{\omega e_{i}}\right\} \mu e_{i} \\
& =-\omega S_{j}(\mu v)+S_{i}(\omega(\mu v))+S_{j}((\omega \mu+\mu \omega) v)=S_{i}(\omega \mu v)+S_{j}(\mu \omega v) \\
& =\left(S_{i}+S_{j}\right)(\omega \mu v)=(\omega \mu) v
\end{aligned}
$$

so that $\{\nu-\omega \mu\} v=0$. Since $\mathfrak{B}$ is faithful by assumption, $\nu=\omega \mu$ :

$$
\left(\omega e_{i}\right)\left(\mu e_{i}\right)=(\omega \mu) e_{i} \text {. }
$$

This implies $\Omega_{i} \Omega_{i} \subset \Omega_{i}$ is a subalgebra, and since $\Omega_{i}$ generates $\Omega$ we get $\Omega_{1}=\Omega_{2}=\Omega$.

For $\omega \in \Omega_{1}=\Omega$ we have $e_{i}\left\{\left(\omega e_{i}\right) \circ v\right\}=\left(\omega e_{i}\right) \circ\left(e_{i} v\right)$ by (13), consequently $S_{i}(\omega v)$ $=\omega S_{i}(v)$, and $S_{i}$ is $\Omega$-linear.

From (15), $P_{i i}(v w)=P_{i i}\left\{\left(e_{i} v\right) \circ w\right\}=Q\left(e_{i} v, w\right) e_{i}$, so if we set $v \times w=P_{12}(v w)$ we get

$$
v w=Q\left(S_{1} v, w\right) e_{1}+v \times w+Q\left(S_{2} v, w\right) e_{2} .
$$

As $P_{i i}(v v)=Q(v) e_{i}$ and $P_{12}(v v)=0$ we know

$$
Q\left(S_{i} v, v\right)=Q(v), \quad v \times v=0 .
$$

Moreover, $v(v \circ w)=v \circ v w$ implies $Q(v, w) v=Q\left(S_{1} v, w\right) v+Q(v, v \times w) 1+Q\left(S_{2} v, w\right) v$ and hence

$$
Q(v, v \times w)=0 .
$$

The product $v \times w$ is $\Omega$-bilinear since $(\omega v) \times w=P_{12}\left\{\left[\left(\omega e_{i}\right) \circ v\right] w\right\}=\left(\omega e_{i}\right) \circ P_{12}(v w)$ (by (17)) $=\omega(v \times w)$,

$$
(\omega v) \times w=\omega(v \times w)=v \times(\omega w)
$$


Finally, the condition $Q(v)=Q\left(S_{i} v, v\right)$ shows that the radical and bilinear radical of $Q$ coincide, so necessarily $Q$ has defect zero.

We denote the algebras constructed in this manner from $Q, S_{i}, \times$ by

$$
\mathfrak{A}=\mathfrak{A}(Q, S, \times) .
$$

6. Radicals. Since we have a notion of invertibility we have a notion of quasiinvertibility: an element $z$ in $\mathfrak{A}$ is quasi-invertible (q.i.) if $1-z$ is invertible in $\mathfrak{A}$ (or $\mathfrak{A}^{\prime}$ if $\mathfrak{A}$ does not have a unit). This is equivalent to the existence of $y \in \mathfrak{A}$ with $(1-z)(1-y)=(1-y)(1-z)=1,(1-z)^{2}(1-y)=(1-y)(1-z)^{2}=1-z$, hence to the intrinsic conditions

$$
z+y=z y=y z \quad z^{2}+z+y=z^{2} y=y z^{2} \quad(\text { or }[z, z, y]=0) .
$$

Since invertibility in $\mathfrak{A}$ coincides with that in $\mathfrak{A}^{+}$, the same is true of quasiinvertibility. We use this to derive results for noncommutative Jordan algebras from those for quadratic Jordan algebras [4]. A subspace is q.i. if all its elements are q.i. The sum of an arbitrary family of q.i. ideals is again a q.i. ideal, so there exists a maximal q.i. ideal called the Jacobson radical $R(\mathfrak{U})$ of the algebra $\mathfrak{A}$. An algebra is semisimple if it has no radical, $R(\mathfrak{U})=0$. If $\mathfrak{B}$ is a q.i. ideal such that $\mathfrak{A} / \mathfrak{B}$ is q.i. then $\mathfrak{A}$ itself is q.i. In particular, $\mathfrak{A} / R(\mathfrak{U})$ contains no q.i. ideals.

THEOREM 10. For any noncommutative Jordan algebra $\mathfrak{A}$ there exists a unique maximal q.i. ideal $R(\mathfrak{A})$, the Jacobson radical, which contains all q.i. ideals. The quotient $\mathfrak{X} / R(\mathfrak{A})$ is semisimple, $R(\mathfrak{U} / R(\mathfrak{U}))=0$.

Since $R(\mathfrak{U})$ is also an ideal in $\mathfrak{A}^{+}$, and its elements are still q.i. in $\mathfrak{A}^{+}$, we see $R(\mathfrak{U})$ is contained in the radical $R\left(\mathfrak{U}^{+}\right)$. Since any subspace of $R\left(\mathfrak{U}^{+}\right)$is q.i. we have the following:

THEOREM 11. The Jacobson radical $R(\mathfrak{H})$ of the noncommutative Jordan algebra $\mathfrak{A}$ is the maximal ideal of $\mathfrak{A}$ which is contained in the Jacobson radical $R\left(\mathfrak{U}^{+}\right)$of the quadratic Jordan algebra $\mathfrak{A}^{+}$.

Examples of algebras satisfying $R(\mathfrak{U})<R\left(\mathfrak{U}^{+}\right)$are the nodal algebras. However, these degree 1 algebras are about the only such examples, for we have

THEOREM 12. If $\mathfrak{A}$ is $n$-interconnected for $n \geqq 2$ then $R(\mathfrak{A})=R\left(\mathfrak{A}^{+}\right)$; in particular, $R(\mathfrak{U})$ contains all trivial elements and $\mathfrak{X} / R(\mathfrak{U})$ is semiprime.

Proof. The last remark follows from the fact [4, p. 676] that $R\left(\mathfrak{U}^{+}\right)$contains all trivial elements of $\mathfrak{A}^{+}$.

If $\mathfrak{A}$ is $n$-interconnected for $n \geqq 2$ we may lump idempotents together and assume $\mathfrak{A}$ is 2-interconnected, $\mathfrak{A}=\mathfrak{U}_{1}+\mathfrak{A}_{1 / 2}+\mathfrak{A}_{0}$ relative to $e \neq 1,0 . R\left(\mathfrak{U}^{+}\right)$is a q.i. subspace containing $R(\mathfrak{U})$, so it will be sufficient if $R\left(\mathfrak{A}^{+}\right)$itslef is an ideal in $\mathfrak{A}$. As an outer ideal, $\Re=R\left(\mathfrak{U}^{+}\right)$has Peirce decomposition $\Re=\Re_{1}+\Re_{1 / 2}+\Re_{0}$.

We claim

(i) $e \Re_{1 / 2} \subset \Re_{1 / 2}$,

(ii) $P_{1 / 2}\left(\mathfrak{A}_{1 / 2} \Re_{1 / 2}\right) \subset \Re_{1 / 2}$. 
To prove an element $z$ belongs to $\Re$ it suffices to show $U_{z} \mathfrak{A} \subset \Re$, since then $\bar{z}$ is trivial in the semisimple algebra $\overline{\mathfrak{A}}^{+}=\mathfrak{A}^{+} / \mathfrak{R}$, implying $\bar{z}=0$. This will be the case for an element $z_{1 / 2} \in \mathfrak{A}_{1 / 2}$ if $P_{i}\left\{z_{1 / 2} \circ \mathfrak{A}_{1 / 2}\right\} \subset \mathfrak{R}_{i}(i=1,0)$ arguing as in Lemma 6. In particular, since $P_{i}\left\{e \mathfrak{R}_{1 / 2} \circ \mathfrak{A}_{1 / 2}\right\}=P_{i}\left\{\mathfrak{R}_{1 / 2} \circ \mathfrak{A}_{1 / 2} e\right\}$ (by (15)) $\subset P_{i}\left(\mathfrak{R}_{1 / 2} \circ \mathfrak{A}_{1 / 2}\right) \subset \mathfrak{R}_{i}$ we have (i), and since

$$
P_{i}\left\{\mathfrak{A}_{1 / 2} \circ P_{1 / 2}\left(\mathfrak{A}_{1 / 2} \mathfrak{R}_{1 / 2}\right)\right\}=P_{i}\left\{\mathfrak{R}_{1 / 2} \circ P_{1 / 2}\left(\mathfrak{A}_{1 / 2} \mathfrak{A}_{1 / 2}\right)\right\} \subset P_{i}\left\{\mathfrak{R}_{1 / 2} \circ \mathfrak{A}_{1 / 2}\right\} \subset \mathfrak{R}_{i}
$$

(by (18)) we have (ii).

These are enough to make $\Re$ an ideal. We have $\mathfrak{A}_{i} \Re_{1 / 2}+\Re_{1 / 2} \mathfrak{A}_{i} \subset \Re_{1 / 2}$ and $\mathfrak{R}_{i} \mathfrak{A}_{1 / 2}+\mathfrak{A}_{1 / 2} \Re_{i} \subset \Re_{1 / 2}$ by (14)' and (i), $\mathfrak{A}_{1 / 2} \Re_{1 / 2}+\mathfrak{R}_{1 / 2} \mathfrak{A}_{1 / 2} \subset \Re$ by (15)' and (ii), and $\mathfrak{A}_{i} \mathfrak{R}_{j}=\mathfrak{R}_{j} \mathfrak{A}_{i}=0$ by orthogonality. It is only for the remaining relation $\mathfrak{A}_{i} \mathfrak{R}_{i}+\mathfrak{R}_{i} \mathfrak{A}_{i} \subset \mathfrak{R}_{i}$ that we need interconnectivity: $\mathfrak{A}_{i}$ is spanned by elements $P_{i}\left(x_{1 / 2}^{2}\right)$, and we apply (20) and (15)'.

REMARK. The same argument shows that the nil radicals (=maximal nil ideals) of $\mathfrak{U}$ and $\mathfrak{U}^{+}$coincide, $N(\mathfrak{U})=N\left(\mathfrak{U}^{+}\right)$, for an $n$-interconnected algebra $(n \geqq 2)$. Indeed, similar results hold for any radical property $\mathbf{P}$ such that a P-semisimple algebra contains no trivial elements.

Proposition 2. If $z \in R(\mathfrak{A})$ is von Neumann regular, $z=U_{z} w$ for some $w$, then $z=0$.

Proof. $z$ is regular in $R\left(\mathfrak{H}^{+}\right)$[4, Theorem 2, p. 673].

The radical $R(\mathfrak{U})$ always contains the nil radical $N(\mathfrak{U})$ since $N(\mathfrak{U})$ is an ideal contained in $N\left(\mathfrak{U}^{+}\right) \subset R\left(\mathfrak{U}^{+}\right)$.

THEOREM 13. If $\mathfrak{A}$ has descending chain condition on inner ideals then $R(\mathfrak{A})$ $=N(\mathfrak{X})$.

Proof. $R(\mathfrak{U}) \subset R\left(\mathfrak{U}^{+}\right)=N\left(\mathfrak{A}^{+}\right)$, where the latter is nil, so $R(\mathfrak{A}) \subset N(\mathfrak{U})[4$, Theorem 13, p. 678].

THEOREM 14. If $\Phi$ is a field and $z \in R(\mathfrak{U})$ is algebraic then $z$ is nilpotent.

Proof. $z \in R\left(\mathfrak{A}^{+}\right)$is algebraic [4, Theorem 3, p. 674].

AMitsUR's TheOREM. If $\Phi$ is a field of cardinality $|\Phi|>2+\operatorname{dim}_{\Phi} \mathfrak{A}$ then $R(\mathfrak{U})$ is nil.

Proof. $R\left(\mathfrak{A}^{+}\right)$is nil by [4, Theorem 4, p. 674].

7. Structure theory. We now impose a finiteness restriction on the algebras we consider-the descending chain condition (d.c.c.) or minimal condition on inner ideals. The Peirce spaces $\mathfrak{A}_{1}$ and $\mathfrak{A}_{0}$ relative to an idempotent $e$ are inner ideals, and it is mainly with such inner ideals that we shall be concerned.

Improving on the methods of [3, pp. 193-194] we may state the

First Structure Theorem. Any semisimple noncommutative Jordan algebra with d.c.c. on inner ideals has a unit and is a direct sum of a finite number of ideals which are themselves simple unital algebras with d.c.c. 
Proof. Let $\mathfrak{C}$ be a minimal ideal in $\mathfrak{A}$ (such exists by the chain condition). Since $\mathfrak{F}$ is not nil (nor is $\mathfrak{C}^{+}$) by semisimplicity, $\overline{\mathfrak{C}}^{+}=\mathfrak{C}^{+} / R\left(\mathfrak{C}^{+}\right)$is a nonzero semisimple quadratic Jordan algebra with d.c.c. and thus [2, p. 1079] contains a unit $\bar{e} \neq 0$. Since $R\left(\mathfrak{C}^{+}\right)$is nil in the presence of the d.c.c. [4, p. 678], we can lift to a principal idempotent $e \neq 0$ for $\mathbb{C}$ (if $x \in \mathbb{E}$ has $\bar{x}=\bar{e}$ then we can find such an $e$ in the commutative associative algebra $\Phi[x]$ by the usual methods). Now by Lemma 1 , $\mathfrak{B}=\mathfrak{B}_{1}+\mathfrak{B}_{1 / 2}+\mathfrak{B}_{0}$ is an ideal in $\mathfrak{A}$ for $\mathfrak{B}_{1 / 2}=\mathfrak{A}_{1 / 2}$ (relative to the idempotent $e$ ), and $\mathfrak{B} \subset \mathfrak{C}$ since $\mathfrak{B}$ is generated by $\mathfrak{A}_{1 / 2}=e \circ \mathfrak{A}_{1 / 2} \subset \mathfrak{C} \circ \mathfrak{A} \subset \mathfrak{C}$. Indeed, since $\mathfrak{A}_{1 / 2}$ $=\mathfrak{夭}_{1 / 2} \subset R\left(\mathfrak{C}^{+}\right)$by the assumption that $\bar{e}$ is the unit for $\overline{\mathfrak{C}}^{+}$, we actually have $\mathfrak{B} \subset R\left(\mathfrak{C}^{+}\right)$. But then $\mathfrak{B}$ is a nil ideal in the semisimple algebra $\mathfrak{A}$, so $\mathfrak{B}=0$. This shows $\mathfrak{A}_{1 / 2}=\mathfrak{B}_{1 / 2}=\mathfrak{V}_{1 / 2}=0$, so that $\mathfrak{A}=\mathfrak{A}_{1} \oplus \mathfrak{A}_{0}$ is a direct sum of ideals. Since $\mathfrak{A}_{1}=e \mathfrak{A}_{1} \subset \mathfrak{C}$, by minimality of $\mathfrak{C}$ we must have $\mathfrak{C}=\mathfrak{A}_{1}$ and $\mathfrak{A}=\mathfrak{C} \oplus \mathbb{C}$. Thus each minimal ideal has a unit and is a direct summand. Since ideals or inner ideals in $\mathfrak{E}=\mathfrak{A}_{1}$ or $\mathfrak{C}^{\prime}=\mathfrak{A}_{0}$ are ideals or inner ideals in $\mathfrak{A}, \mathfrak{C}^{\prime}$ is again semisimple with d.c.c. on inner ideals and $\mathfrak{C}$ is simple, unital, with d.c.c. Repeating the procedure to $\mathfrak{C}^{\prime}$, and noting that the process must eventually terminate since it produces a decreasing sequence of ideals, we see $\mathfrak{A}$ is a finite direct sum of simple ideals $\mathfrak{E}_{\mathfrak{i}}$ having units $e_{i}$ and d.c.c. In particular, $\mathfrak{A}$ itself has unit $1=\sum e_{i}$.

Right away we can say the simple summands are either of degree 1,2 , or $n \geqq 3$ (the degree being the maximal number of orthogonal idempotents in the algebra, rather than in extensions of the algebra), and by the general Theorem 5 those of degree $n \geqq 3$ are commutative or quasiassociative. Furthermore, by Theorem 12 those summands of degree $\geqq 2$ are semiprime. In the presence of the d.c.c. we can describe these degree $\geqq 2$ algebras in more detail. However, we cannot apply our Coordinatization Theorems or our knowledge of the structure of $\mathfrak{A}^{+}$immediately-in the quadratic Jordan case we can only conclude that an isotope $\mathfrak{A}^{+(u)}$ of $\mathfrak{A}^{+}$has a capacity, not $\mathfrak{A}^{+}$itself, and it is not clear that $\mathfrak{A}^{+(u)}$ still carries a suitable noncommutative structure. (This passage to an isotope only comes up in characteristic 2.) What we shall do is go back through the process leading up to the classification of quadratic Jordan algebras and show that for those semiprime algebras of the special form $\mathfrak{A}^{+}$the passage to an isotope is unnecessary, that we already have a rich supply of good idempotents in $\mathfrak{A}$.

An idempotent $e$ is completely primitive if the Peirce space $\mathfrak{A}_{1}(e)$ is a division algebra. The result on which everything hinges is the

Minimal InNer Ideal Theorem. A minimal inner ideal $\mathfrak{B}$ in a noncommutative Jordan algebra $\mathfrak{A}$ is one of the following three types:

I. $\mathfrak{B}=\Phi z$ for $z$ trivial;

II. $\mathfrak{B}=U_{b} \mathfrak{A}$ for all $b \neq 0$ in $\mathfrak{B}$ but $\mathfrak{B}^{2}=U_{\mathfrak{B}} \mathfrak{B}=0$;

III. $\mathfrak{B}=U_{e} \mathfrak{A}$ for some completely primitive idempotent $e$.

In type II there exists an idempotent $e \neq 1,0$ in $\mathfrak{A}$ (though not in $\mathfrak{B})$.

Proof. From the quadratic Jordan case $[2$, p. 1078] we know any minimal $\mathfrak{B}$ in 
$\mathfrak{\Im}=\mathfrak{A}^{+}$is one of I-III. For a general $\mathfrak{\Im}$ the type II case need not lead to an idempotent $e \neq 1,0$, though such $e$ exists in an isotope of $\mathfrak{\Im}$; we want to show if $\mathfrak{\Im}=\mathfrak{A}^{+}$ has the additional noncommutative structure there must exist an $e \neq 1,0$ in $\Im$ itself.

Suppose $\mathfrak{A}$ does not contain $e \neq 1,0$. Again by the Jordan theory there is a nonzero $b \in \mathfrak{B}$ and a $d \in \mathfrak{A}$ with

$$
b^{2}=d^{2}=0, \quad b \circ d=1, \quad U_{b} d=b, \quad U_{d} b=d
$$

(so $\mathfrak{A}$ is necessarily unital), and the characteristic is 2 .

The prototype for this situation is $\Im=\Phi_{2}^{+}, b=e_{12}, d=e_{21}$. If we are in the quasiassociative algebra $\mathfrak{A}=\Phi_{2}^{(\lambda)}$ then $x=b \cdot{ }_{\lambda} d=\lambda e_{11}+(1-\lambda) e_{22}$ has $x^{2}-x=z=-\varphi 1$ for $\varphi=\lambda(1-\lambda)$. In this case $e=b-z \cdot{ }_{\lambda} d+x=\left(\begin{array}{cc}\lambda & 1 \\ \varphi & -\lambda\end{array}\right)$ is an idempotent $\neq 1,0$.

This leads us to define in general

$$
e=b-z d+x, \quad x=b d, \quad z=x^{2}-x,
$$

and hope $e$ turns out to be the desired idempotent. We first need some information about $x$. From $b=L_{b} 1=L_{b} V_{b} d=V_{b} L_{b} d$ we get $b=b \circ x$, and similarly for $d=R_{d} 1$, so

$$
b \circ x=b, \quad d \circ x=d .
$$

Whenever $a \circ x=a$ we get $0=\left[L_{x^{2}}, E_{a}\right]+\left[L_{x \circ a}, E_{x}\right]=\left[L_{x^{2}}, E_{a}\right]+\left[L_{a}, E_{x}\right]=\left[L_{x^{2}}, E_{a}\right]$ $-\left[L_{x}, E_{a}\right]=\left[L_{z}, E_{a}\right]$ from (3), $L_{z}$ commutes with $E_{a}$ if $a \circ x=a(E=L, R, V)$. This applies to $a=b, d$ by the above, and also to $a=z d$ since $x \circ z d=V_{x} L_{z} d=L_{z} V_{x} d$ (remember $z=x^{2}-x$, so use (9)) $=L_{z} d=z d$. The commutativity of $L_{z}$ with $V_{x}$ and $V_{b}$ gives $b \circ z d=z(b \circ d)$ and $x \circ z d=z(x \circ d)$, while commutativity with $R_{z d}$ and $L_{d}$ gives

$$
(z d)^{2}=[z, d, z d]+z\{d(z d)\}=-\left[L_{z}, R_{z d}\right] d+z\left\{L_{d} L_{z} d\right\}=0+z\left\{L_{z} L_{d} d\right\}=0 .
$$

Thus

$$
b \circ z d=z, \quad x \circ z d=z d, \quad(z d)^{2}=0 .
$$

We compute

$$
\begin{aligned}
e^{2} & =b^{2}+(z d)^{2}+x^{2}-b \circ(z d)-x \circ(z d)+b \circ x \\
& =0+0+(x+z)-z-z d+b \\
& =b-z d+x=e
\end{aligned}
$$

so $e$ is idempotent. We have

$$
\begin{aligned}
e \circ d & =b \circ d-z(d \circ d)+x \circ d \quad\left(L_{z} \text { commutes with } V_{d}\right) \\
& =1+d
\end{aligned}
$$

so $e \neq 0$ because $d \neq-1$ and $e \neq 1$ because $d \neq 1$. Thus we have our desired $e \neq 1,0$.

Lemмa 11. If the semiprime noncommutative Jordan algebra $\mathfrak{A}$ has the d.c.c. on inner ideals then any idempotent in $\mathfrak{A}$ is a sum of primitive idempotents, and any primitive idempotent is completely primitive. 
Proof. That any idempotent is a sum of primitive idempotents in the presence of the d.c.c. is true in any quadratic Jordan algebra, hence in $\mathfrak{A}^{+}$and in $\mathfrak{A}$. If $e$ is primitive then $\mathfrak{A}_{1}(e)$ has no idempotents except $e=1$ and 0 , and if $\mathfrak{A}$ is semiprime with d.c.c. so is $\mathfrak{A}_{1}(e)$. Thus there can be no minimal inner ideals of type I or II in $\mathfrak{A}_{1}(e)$, and there exists a unique minimal inner ideal of type III, namely $U_{e} \mathfrak{A}=\mathfrak{A}_{1}(e)$ itself with $e$ completely primitive.

We have already seen that any semisimple noncommutative Jordan algebra has a unit; we now give an alternate proof of this for the less general case of semiprime algebras. We say an algebra has capacity $n$ if it has a unit which is the sum of $n$ orthogonal completely primitive idempotents.

THEOREM 15. A semiprime noncommutative Jordan algebra with descending chain condition on inner ideals has a capacity.

Proof. By Lemma 11 we need only show $\mathfrak{A}$ has a unit. Choose a maximal $e$ (i.e. a minimal $\left.\mathfrak{A}_{0}(e)\right) ; \mathfrak{A}_{0}(e)$ inherits semiprimeness and the d.c.c., so by the Minimal Inner Ideal Theorem if it is nonzero it contains an idempotent $f \neq 0$ contradicting the maximality of $e: \mathfrak{A}_{0}(e+f)<\mathfrak{A}_{0}(e)$ since $f \in \mathfrak{A}_{0}(e), f \notin \mathfrak{A}_{0}(e+f)$. We must therefore have $\mathfrak{A}_{0}=0$, and, by Lemma $6, \mathfrak{A}=\mathfrak{A}_{1}$ has unit $e$.

Now we are ready to state the

Second Structure Theorem. A simple noncommutative Jordan algebra with descending chain condition on inner ideals is isomorphic to one of the following:

I. a degree 1 algebra in which every element is either invertible or nilpotent;

II. a split algebra $\mathfrak{A}(Q, S, \times)$ determined by a nondegenerate quadratic form $Q$ of defect 0 on a vector space $\mathfrak{B}, S: \mathfrak{B} \rightarrow \mathfrak{B}$ a linear transformation such that $Q(S v, v)=Q(v)$, and a bilinear product on $\mathfrak{B}$ such that $v \times v=0$ and $Q(v \times w, v)=0$;

III. a commutative Jordan matrix algebra $\mathfrak{S}_{\mathfrak{C}}\left(\mathfrak{D}_{n}, \gamma\right)$ of characteristic $\neq 2$ for $n \geqq 2$, D either (i) a direct sum $\mathfrak{D}=\Delta \oplus \Delta^{0}$ of anti-isomorphic associative division algebras with exchange involution, (ii) an associative division algebra $\mathfrak{D}=\Delta$ with involution, (iii) $\mathfrak{D}=\Omega_{2}$ a split quaternion algebra over its center (only for $n \geqq 3$ ), or (iv) $\mathfrak{D}=\mathbb{C} a$ Cayley algebra (only for $n=3$ );

IV. a quasiassociative algebra $\mathfrak{S}_{(}\left(\mathfrak{D}_{n}, \gamma\right)^{(\lambda)}$ where $n \geqq 2, \mathfrak{D}=\Delta \oplus \Delta^{0}$ or $\mathfrak{D}=\Delta$ for $\Delta$ an associative division algebra, and $\lambda \neq \frac{1}{2}$ a central element of $\mathfrak{D}$ with $\lambda+\lambda=1$.

Proof. First assume $\mathfrak{A}$ has degree $\geqq 2$. By Theorem $12, \mathfrak{A}$ is semiprime, so by Theorem $15, \mathfrak{A}^{+}$has a capacity $n \geqq 2$; since $\mathfrak{A}^{+}$is simple by Theorem 1 , we know [2, p. 1079] it must be either

(i) a split algebra $\operatorname{Sp}\left(Q, \Omega_{1}, \Omega_{2}\right)$ of a nondegenerate quadratic form $Q$ on a vector space $\mathfrak{B}$ over a field $\Omega$, where the subspaces $\Omega_{i}$ contain 1 and $Q(\mathfrak{B}) \Omega_{j}$ and where the $\Omega_{i}$ generate $\Omega$;

(ii) a Jordan matrix algebra $\mathfrak{S}_{(}\left(\mathfrak{D}_{2}, \mathfrak{D}_{0}, \gamma\right)$ where $\mathfrak{D}=\Delta \oplus \Delta^{0}$ or $\mathfrak{D}=\Delta$ for $\Delta$ an associative division algebra, and where $\mathfrak{D}_{0}$ generates $\mathfrak{D}$; 
(iii) a Jordan matrix algebra $\mathfrak{S}_{(}\left(\mathfrak{D}_{n}, \mathfrak{D}_{0}, \gamma\right)$ for $n \geqq 3$ where either (1) $\mathfrak{D}=\Delta \oplus \Delta^{0}$ for $\Delta$ an associative division algebra, (2) $\mathfrak{D}=\Delta$ an associative division algebra, (3) $\mathscr{D}=\Omega_{2}$ a split quaternion algebra, (4) $\mathfrak{D}=\mathbb{C}$ a Cayley algebra (only for $n=3$ ).

If $\mathfrak{A}^{+}=\operatorname{Sp}\left(Q, \Omega_{1}, \Omega_{2}\right)$ has capacity 2 then, by Theorem $9, \mathfrak{A}=\mathfrak{A}(Q, S, \times)$ as in case II. If $\mathfrak{A}^{+}=\mathfrak{S}\left(\mathfrak{D}_{2}, \mathfrak{D}_{0}, \gamma\right)$ has capacity 2 as in (ii) then by Theorem 8 we have either case III or IV with $n=2$. If $\mathfrak{A}^{+}=\mathfrak{S}\left(\mathfrak{D}_{n}, \mathfrak{D}_{0}, \gamma\right)$ has capacity $n \geqq 3$ as in (iii) then by the Coordinatization Theorem we have either III or IV (note that in (iii) the composition algebras (3) and (4) cannot lead to quasiassociative algebras $\mathfrak{S}\left(\mathfrak{D}_{n}, \gamma\right)^{(\lambda)}$ since $\Omega_{2}$ and $\mathbb{E}$ do not contain $\lambda \neq \frac{1}{2}$ in their center with $\lambda+\lambda=1$; the centers are fixed under the involution).

Now consider the degree 1 case. Here $\mathfrak{A}$ has no idempotents except $e=1,0$. That all elements are invertible or nilpotent as in I then follows by applying the following general proposition (due to D. Morgan) to $\mathfrak{\Im}=\mathfrak{U}^{+}$:

PROPOSITION 3. If $\Im$ is a quadratic Jordan algebra with d.c.c. on inner ideals which contains no idempotents $e \neq 1,0$ then every element in $\Im$ is either invertible or nilpotent.

Proof. Suppose $x \in \mathfrak{I}$ is not nilpotent. By the d.c.c. the chain of inner ideals $U_{x \mathfrak{l}} \supset U_{x^{2}} \mathfrak{} \supset \ldots \supset U_{x^{n}} \mathfrak{\Im}=U_{x^{n+1}} \mathfrak{\Im}=\cdots$ breaks off at some stage. If we set $y=x^{n+1}$ then $y^{2}$ is regular, $y^{2}=U_{y^{2}} a$, since $y^{2}=x^{2 n+2}=U_{x^{n}} x^{2} \in U_{x^{n}} \mathfrak{\Im}=U_{x^{2 n+2}} \mathfrak{~ . ~ N o w ~ a n y ~}$ time $y^{2}=U_{y^{2}} a$ the element $e=U_{y} U_{a} y^{2}$ is idempotent because

$$
\begin{aligned}
e^{2} & =U_{y} U_{a} U_{y^{2}} U_{a} y^{2}=U_{y} U_{a} U_{y^{2}} U_{a}\left(U_{y^{2}} a\right)=U_{y} U_{a}\left(U_{U\left(y^{2}\right) a}\right) a \\
& =U_{y} U_{a} U_{y^{2}} a=U_{y} U_{a} y^{2}=e .
\end{aligned}
$$

In our case $y^{4}=x^{4 n+4}$ is not zero since $x$ is not nilpotent, and whenever $y^{4} \neq 0$ the above $e$ is also nonzero: the formula $\left(U_{z} w\right)^{2}=U_{z} U_{w} z^{2}$ [2, p. 1073] shows $U_{y} e$ $=U_{y^{2}} U_{a} y^{2}=\left(U_{y^{2}} a\right)^{2}=\left(y^{2}\right)^{2}=y^{4}$. Since $e \neq 0$ the only other idempotent it could be is $e=1$. Since $1=e \in U_{y} \Im \subset U_{x} \mathfrak{\jmath}$, we see $x$ is invertible.

Further insight into quadratic Jordan algebras of degree 1 is given by

Proposition 4. $\Im$ is a semisimple quadratic Jordan algebra with d.c.c. on inner ideals which contains no idempotents $e \neq 0,1$ if and only if $\mathfrak{\Im}$ is either (i) a Jordan division algebra, or (ii) an isotope $\operatorname{Sp}(Q)^{(v)}$ of a split algebra $\Omega_{0} e_{1}+\Omega_{0} e_{2}+\mathfrak{B}$ of a quadratic form on $\mathfrak{B}$ of defect $\geqq 1, Q(v, \mathfrak{B})=0$.

Proof. By the Structure Theory [2], $\Im$ is a direct sum of ideals which are unital simple algebras with d.c.c. Since $\mathfrak{\Im}$ has only one nonzero idempotent, it has only one simple component, and $\mathfrak{\Im}$ itself is simple. Since it is semisimple it has no inner ideals of type I, and none of type III unless it is a division algebra. Otherwise all its minimal inner ideals must be of type II; in particular, $\Im$ has characteristic 2 and some isotope $\mathfrak{F}^{(u)}$ is simple and strongly connected of capacity 2 . By Osborn's Theorem [2], such a strongly connected algebra of capacity 2 is isomorphic to one of the following: 
(i) $\Delta_{2}^{+}$for $\Delta$ an associative division algebra;

(ii) $\mathfrak{S}_{2}\left(\Delta_{2}, \Delta_{0}\right)$ for $\Delta$ an associative division algebra with nontrivial involution, $\Delta_{0}$ an ample subspace;

(iii) a split algebra $\operatorname{Sp}(Q)=\Omega_{0} e_{1}+\Omega_{0} e_{2}+\mathfrak{B}$ determined by a quadratic form $Q$ on $\mathfrak{B}$.

The original algebra $\Im=\left\{\Im^{(u)}\right\}^{\left(u^{-2}\right)}$ is thus an isotope of an algebra of the form (i)-(iii). Any isotope of $\Delta_{2}^{+}$is isomorphic to $\Delta_{2}^{+}$, hence has nontrivial idempotents. $\mathfrak{S}\left(\Delta_{2}, \Delta_{0}\right)$ is isomorphic to the algebra of all linear transformations $x$ on a 2dimensional vector space $\mathfrak{B}$ over $\Delta$ which are selfadjoint relative to a nondegenerate nonalternate Hermitian bilinear form $\langle v, w\rangle$ on $\mathfrak{B}(\langle x(v), w\rangle=\langle v, x(w)\rangle)$ which take quadratic values $\langle x(v), v\rangle$ in $\Delta_{0}$. Any isotope of such an algebra has the same form (relative to a new Hermitian bilinear form $\langle v, w\rangle^{\prime}$ which is nonalternate unless $\Delta$ is a field of characteristic 2 with identity involution); since a nonalternate form can be diagonalized we can find nontrivial idempotents. The only remaining possibility is $\Im=\operatorname{Sp}(Q)^{(v)}$. If $\widetilde{Q}\left(\alpha_{1} e_{1}+\alpha_{2} e_{2}+v\right)=\alpha_{1} \alpha_{2}+Q(v)$ then the operations in $\operatorname{Sp}(Q)$ are given by $U_{x} y=\widetilde{Q}\left(x, y^{*}\right) x-\widetilde{Q}(x) y^{*}\left(y^{*}=\widetilde{T}(y) 1-y\right)$, so the square in $\operatorname{Sp}(Q)^{(v)}$ is $x^{2(v)}=U_{x} v=\widetilde{Q}\left(x, v^{*}\right) x-\widetilde{Q}(x) v^{*}$. We will show that if $\operatorname{Sp}(Q)^{(v)}$ has no proper idempotents then $v \in \mathfrak{B}$ and $Q(v, \mathfrak{B})=0$ so $v$ is in the bilinear radical of $Q$; since the radical of $Q$ is zero if $\operatorname{Sp}(Q)$ is simple, this will show the defect of $Q$ is $\geqq 1$. If $x \neq 0$ but $\widetilde{Q}(x)=0$ then $x \neq 0,1^{(v)}$ so $x$ cannot be idempotent in $\Im$ by hypothesis: $x^{2(v)}=\widetilde{Q}\left(x, v^{*}\right) x \neq x$. This implies $\widetilde{Q}\left(x^{*}, v\right)=\widetilde{Q}\left(x, v^{*}\right)=0$ whenever $\widetilde{Q}(x)=0$. Taking $x=e_{1}, x=e_{2}$, we see $v \in \mathfrak{B}=\left(\Omega_{0} e_{1}+\Omega_{0} e_{2}\right)^{\perp}$; for any $w \in \mathfrak{B}$ we take $x=e_{1}-Q(w) e_{2}+w$, so $\widetilde{Q}(x)=0$, hence $0=\widetilde{Q}(x, v)=Q(w, v)$, and $Q(v, \mathfrak{B})=0$ as claimed.

Conversely, one can easily verify that the 2 possibilities actually do furnish degree 1 algebras.

8. Degree 1 algebras. We close with a few remarks about central simple algebras of degree 1, those having no idempotents except $e=1$ and $e=0$.

Suppose $\mathfrak{A}$ has degree 1 and has the d.c.c. By the Minimal Inner Ideal Theorem, a degree 1 algebra has no inner ideals of type II, and it has inner ideals of type III if and only if it is a division algebra.

Thus a degree 1 algebra with d.c.c. has a capacity if and only if it is a division algebra. Conversely, any division algebra has capacity 1 and trivially has the d.c.c. since there are no proper inner ideals. Thus the d.c.c. does not serve as a finiteness restriction, and there is little we can say about arbitrary noncommutative (or even quadratic) Jordan division algebras.

Suppose now that $\mathfrak{A}$ has no capacity. Then all its minimal inner ideals must be trivial of type $\mathrm{I}$. We claim any scalar extension of such a central simple algebra is again of the same type. Indeed, if $\mathfrak{A}$ has trivial elements so does any extension $\mathfrak{A}_{\Omega}$ (if $U_{z} \mathfrak{A}=0$ then also $U_{z} \mathfrak{A}_{\Omega}=0$ and $z$ stays trivial in $\mathfrak{A}_{\Omega}$ ). By Theorems 1,12 this implies the central simple algebra $\mathfrak{A}_{\Omega}$ has no idempotents $e \neq 1,0$, so $\mathfrak{A}_{\Omega}$ remains of degree 1 without capacity. 
If in addition we assume $\mathfrak{A}$ is finite dimensional over its center $\Phi$ and take $\Omega$ to be the algebraic closure of $\Phi$, we claim $\mathfrak{A}_{\Omega}$ is a nodal algebra in the sense that every element has the form $a=\alpha 1+z$ for some $\alpha \in \Omega$ and some nilpotent $z$, but the nilpotent elements do not form an ideal. Indeed, since $\mathfrak{A}_{\Omega}$ contains no idempotents $e \neq 1,0$ the minimum polynomial of $a \in \mathfrak{A}_{\Omega}$ cannot have distinct roots: $\mu_{a}(t)$ $=(t-\alpha)^{k}$. Then $z=a-\alpha 1$ is nilpotent, $z^{k}=0$. Nilpotent elements exist since $\mathfrak{A}_{\Omega}$ is not a division algebra, yet not all elements are nilpotent, so the nilpotent elements cannot form an ideal in the simple algebra $\mathfrak{A}_{\Omega}$.

An algebra is almost nil if it has the form $\mathfrak{U}=\Phi 1+\mathfrak{R}$ for $\mathfrak{R}$ a nil subspace (necessarily an ideal in $\mathfrak{A}^{+}$). An almost nil algebra is nodal if $\mathfrak{N}$ does not form an ideal in $\mathfrak{A}$. It is known that nodal algebras exist only for characteristic $p \neq 0$, and for $p \neq 2$ they are necessarily almost nil and their structure is more or less known $[11$, p. 144].

In characteristic 2 it is known that a nodal quadratic Jordan algebra need not be almost nil. However, we conjecture that even in characteristic 2 a nodal noncommutative Jordan algebra (with d.c.c.) is almost nil. (We have already seen a situation-the Minimal Inner Ideal Theorem-where the extra structure in the noncommutative case gave us a stronger result than in the quadratic case.)

For almost nil algebras we have

THEOREM 16. If $\mathfrak{A}=\Phi 1+\mathfrak{N}$ is a simple noncommutative Jordan algebra over a field $\Phi$ of characteristic 2 , for $\mathfrak{R}$ a nil subspace, then $\mathfrak{A}$ is commutative and has multiplication

$$
z w=\lambda(z, w) 1+z \times w \quad(z, w \in \mathfrak{R})
$$

where $\lambda$ is an alternate bilinear form and $\times$ an alternate bilinear product on $\mathfrak{R}$,

$$
\lambda(z, z)=0 \quad z \times z=0 .
$$

Conversely, any algebra $\mathfrak{A}=\Phi 1+\mathfrak{N}$ built up in this manner from an alternate algebra $(\mathfrak{R}, \times), z \times z=0$, and an alternate form $\lambda, \lambda(z, z)=0$, is a noncommutative Jordan algebra; it will be nodal if and only if $\lambda \neq 0$, and simple if and only if $\operatorname{Rad} \lambda$ contains no ideals of $(\mathfrak{N}, \times)$.

Proof. By Lemma 5 we know $\{\mathfrak{A} \mathfrak{A} \mathfrak{A}\}=\{\mathfrak{R} \mathfrak{N} \mathfrak{R}\}+\mathfrak{N} \circ \mathfrak{N}+2 \mathfrak{N}+2 \Phi 1$ is an ideal in $\mathfrak{A}=\Phi 1+\mathfrak{N}$. We have $U_{\mathfrak{R}} \mathfrak{N}$ and $\mathfrak{N}^{2}$ contained in $\mathfrak{N}$, since if $U_{z} w$ or $z^{2}$ were not in $\mathfrak{R}$ it would be invertible, implying $z \in \mathfrak{N}$ is invertible, whereas $z$ is nilpotent. Since $\mathfrak{R}$ is a subspace we can linearize to get $\{\mathfrak{R} \mathfrak{R} \mathfrak{R}\}$ and $\mathfrak{R} \circ \mathfrak{R}$ contained in $\mathfrak{N}$. Thus in characteristic 2, $\{\mathfrak{U} \mathfrak{A} \mathfrak{A}\} \subset \mathfrak{N}<\mathfrak{A}$. By simplicity $\{\mathfrak{U} \mathfrak{A} \mathfrak{A}\}=0$. In particular, $\mathfrak{A} \circ \mathfrak{A}=0$, and $x y+y x=x \circ y=0$ means $x y=y x$, therefore that $\mathfrak{A}$ is commutative.

In a commutative algebra of characteristic 2 ,

$$
\left[x^{2}, y, z\right]=\left[x^{2}, y, z\right]+[x \circ z, y, x]=0
$$

(using the commutativity and formula (2)'), so that $L_{x^{2}}$ commutes with all $L_{z}=R_{z}$. This means $x^{2}$ lies in the center of $\mathfrak{A}$. Thus $x$ is nilpotent if and only if $x^{2}=0$, and $\mathfrak{N}=\left\{z \mid z^{2}=0\right\}$. If we let

$$
z w=\lambda(z, w) 1+z \times w
$$


for $\lambda(z, w) \in \Phi$ and $z \times w \in \mathfrak{N}$ when $z, w$ belong to $\mathfrak{R}$ then $z^{2}=0$ shows

$$
\lambda(z, z)=0 \quad z \times z=0 .
$$

Conversely, if $\mathfrak{A}$ is constructed from $\lambda$ and $\times$ in this way then $z^{2}=0$ for all $z$ in $\mathfrak{R}$, so $a^{2}=(\alpha 1+z)^{2}=\alpha^{2} 1+z^{2}=\alpha^{2} 1$ trivially implies $\left[a^{2}, b, a\right]=0$ for all $a, b$ in the algebra. Also $\lambda(z, w)=-\lambda(w, z)=\lambda(w, z), w \times z=-z \times w=z \times w$ are both symmetric, which shows $\mathfrak{A}$ is commutative and therefore trivially flexible: $[a, b, a]$ $=0$. Thus $\mathfrak{A}$ is a noncommutative Jordan algebra. An ideal $\mathfrak{B}$ is proper if and only

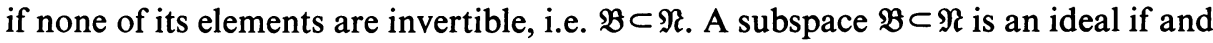
only if $\mathfrak{B R} \subset \mathfrak{B}$, i.e.

$$
\lambda(\mathfrak{B}, \mathfrak{R})=0 \quad \mathfrak{B} \times \mathfrak{R} \subset \mathfrak{B},
$$

which means that $\mathfrak{B}$ is an ideal of $\mathfrak{R}$ contained in $\operatorname{Rad} \lambda$.

We denote algebras built up from $\lambda$ and $\times$ like this by

$$
\mathfrak{A}(\lambda, \times) \text {. }
$$

Simple algebras $\mathfrak{A}(\lambda, \times)$ are easy to come by (and therefore hard to classify). For example, a nondegenerate form $\lambda$ automatically leads to a simple algebra no matter what the product $\times$ is. Similarly, a simple algebra $\mathfrak{N}$ with $z \times z=0$ (for example, a simple Lie algebra) always leads to a simple algebra $\mathfrak{A}$ as long as $\lambda \neq 0$ is not identically zero.

We might add that if a noncommutative Jordan algebra of characteristic 2 is restricted [10] in the sense that $L_{x^{2}}=L_{x}^{2}$ then by $(1)^{\prime} R_{x^{2}}=R_{x}^{2}$ too, so $\mathfrak{A}$ is actually alternative. It is known that there are no nodal alternative algebras.

Finally, these almost nil algebras show that a noncommutative Jordan algebra which happens to be commutative (a "commutative" Jordan algebra) is a very different beast in characteristic 2 from a (quadratic) Jordan algebra.

\section{REFERENCES}

1. A. A. Albert, Power associative rings, Trans. Amer. Math. Soc. 64 (1948), 552-593. MR 10, 97.

2. K. McCrimmon, A general theory of Jordan rings, Proc. Nat. Acad. Sci. U.S.A. 56 (1966), 1072-1079. MR 34 \#2643.

3. - Structure and representations of noncommutative Jordan algebras, Trans. Amer. Math. Soc. 121 (1966), 187-199. MR 32 \#5700.

4. - The radical of a Jordan algebra, Proc. Nat. Acad. Sci. U.S.A. 62 (1969), 671-678.

5. - - A note on quasi-associative algebras, Proc. Amer. Math. Soc. 17 (1966), 1455-1459. MR 39 \#270.

6. - Norms and noncommutative Jordan algebras, Pacific J. Math. 15 (1965), 925-956. MR 34 \#4317.

7. - Quadratic Jordan algebras and cubing operations, Trans. Amer. Math. Soc. 153 (1971), 265-278.

8. K. McCrimmon and R. D. Schafer, On a class of noncommutative Jordan algebras, Proc. Nat. Acad. Sci. U.S.A. 56 (1966), 1-4. MR 34 \#5888. 
9. R. D. Schafer, Noncommutative Jordan algebras of characteristic 0, Proc. Amer. Math. Soc. 6 (1955), 472-475. MR 17, 10.

10. - Restricted noncommutative Jordan algebras of characteristic p, Proc. Amer. Math. Soc. 9 (1958), 141-144. MR 21 \#2678.

11. - An introduction to nonassociative algebras, Pure and Appl. Math., vol. 22, Academic Press, New York, 1966. MR 35 \#1643.

12. K. C. Smith, Noncommutative Jordan algebras of capacity 2 (to appear).

13. N. Jacobson, Lectures on quadratic Jordan algebras, Tata Institue Lecture Notes, Bombay, 1969.

14. R. E. Lewand and K. McCrimmon, Macdonald's theorem for quadratic Jordan algebras, Pacific J. Math. (1971).

UNIVERSITY OF VIRGINIA,

Charlottesville, Virginia 22901 